\title{
Analysis of the Properties of Clusters of Galaxies in the Region of the Ursa Major Supercluster
}

\author{
F. G. Kopylova ${ }^{1}$ and A. I. Kopylov ${ }^{1}$ \\ ${ }^{1}$ Special Astrophysical Observatory of the Russian AS, Nizhnij Arkhyz 369167, Russia
}

(Received August 20, 2008; Revised September 25, 2008)

\begin{abstract}
We analyze the properties of the clusters of galaxies in the region of the Ursa Major (UMa) supercluster using observational data from SDSS and 2MASS catalogs. The region studied includes a supercluster (with a galaxy and cluster overdensity of 3 and 15, respectively) and field clusters inside the $150-\mathrm{Mpc}$ diameter surrounding region. The total dynamical mass of 10 clusters of galaxies in UMa is equal to $2.25 \times 10^{15} M_{\odot}$, and the mass of 11 clusters of galaxies in the UMa neighborhood is equal to $1.70 \times 10^{15} M_{\odot}$. The fraction of early-type galaxies brighter than $M_{K}^{*}+1$ in the virialized regions of clusters is, on the average, equal to $70 \%$, and it is virtually independent on the mass of the cluster. The fraction of these galaxies and their average photometric parameters are almost the same both for UMa clusters and for the clusters located in its surroundings. Parameters of the clusters of galaxies, such as infrared luminosities up to a fixed magnitude, the mass-to-luminosity ratio, and the number of galaxies have almost the same correlations with the cluster mass as in other samples of galaxies clusters. However, the scatter of these parameters for UMa member clusters is twice smaller than the corresponding scatter for field clusters, possibly, due to the common origin of UMa clusters and synchronized dynamical evolution of clusters in the supercluster.
\end{abstract}

\section{INTRODUCTION}

Clusters of galaxies, which are the biggest virialized systems in the Universe, form even larger structures-the superclusters. Superclusters consist of two to twenty clusters and groups of galaxies, which are located either in filaments or at the intersections of filaments (nodes). Superclusters are large structures and their study requires the use of extensive observational data-first and foremost, radial velocities of galaxies. For example, based on their analysis of the $2 \mathrm{dF}$ survey, Einasto et al. [1] revealed the following property of the superclusters of galaxies: rich clusters of galaxies, located in the densest regions of rich superclusters, contain greater fractions of early-type galaxies. Note that the brightest galaxies of the main clusters (i.e., bright clusters located near the peak of luminosity density) in rich superclusters are brighter than the corresponding galaxies in poor superclusters or field groups. An analysis of the rich supercluster Corona Borealis $(z \sim 0.07,[2])$ shows that this system exhibits an excess of bright galaxies compared to the field. A study of the 
properties of galaxies in the Shapley supercluster yielded the following results [3]: the $(B-R, R)$ color-magnitude relation shows that early-type galaxies in the cores of clusters are 0.015 redder (older) than in less dense regions, whereas the fraction of late-type galaxies increases with decreasing local galaxy density and with increasing magnitude. Moreover, the Schechter function fits poorly the composite luminosity function of the supercluster [4].

The aim of this paper is to compare the properties of clusters of galaxies and the average parameters of the subsamples located within the virial radius: (1) 12 clusters located in the Ursa Major supercluster and (2) 12 isolated field clusters located in the nearest neighborhood of the Ursa Major supercluster within $75 \mathrm{Mpc}$ of the center of the system. For this study we used the data of the 2MASS (Two-Micron All-Sky Survey) and SDSS (Sloan Digital Sky Survey) catalogs. The paper has the following layout. Part 2 describes the parameters of clusters of galaxies: the dispersion of galaxy velocities, radius of the virial region, and mass. In Part 3 we compute and list the total $K_{s}$-band (hereafter simply referred to as $K$-band) luminosities of the clusters, the composite luminosity function of the clusters of the system, the luminosity functions of early- and late-type galaxies, and various correlations between the parameters of clusters of galaxies. In Conclusions we list the results obtained. Throughout this paper we adopt the following cosmological parameters: $\Omega_{m}=0.3, \Omega_{\Lambda}=0.7$, and $H_{0}=70 \mathrm{~km} / \mathrm{s} / \mathrm{Mpc}$.

\section{PARAMETERS OF CLUSTERS}

The sample studied is made up of 12 member clusters of the UMa supercluster (the supercluster is the space region where the galaxy overdensity is about 3 [5]), and 12 clusters of galaxies located in the UMa neighborhood with a lower overdensity. The redshifts of clusters lie in the interval $0.045<z<0.075$. We use for the galaxies studied the spectroscopic data reported in the SDSS (Data Release 5) catalog and supplement them by the data adopted from NED. In our earlier papers we used the Data Release 3 [6] and Data Release 4 [5] catalogs, which contained incomplete data for some of the clusters (A1291, A1377, A1436, and Anon4). In this paper we refine all the parameters of the clusters of galaxies based on SDSS data (our previous papers were based on the 2MASS catalog). 


\subsection{Dynamic Parameters of Clusters of Galaxies}

We determine the dynamical masses of clusters from the dispersion of galaxy radial velocities assuming that the cluster is in virial equilibrium and the masses of galaxies increase linearly with radius. According to Carlberg et al. [7], the radius of a cluster whose density is 200 times higher than the critical density, is close to virial radius and can be estimated by the formula $R_{200}=$ $\sqrt{3} \sigma /(10 H(z))$ Mpc. The mass within $R_{200}$ is equal to $M_{200}=3 G^{-1} R_{200} \sigma_{200}^{2}$. We thus first estimate the average radial velocity $c z$ of the cluster and its dispersion $\sigma$, and then find the $R_{200}$ radius from the dispersion. We consider the galaxies with velocities greater than $2.5 \sigma$ to be background objects. We use the iteration method to find all the cluster parameters within the given radius.

We summed up the resulting masses of 10 clusters in UMa (with the exception of A1291B and Anon2 clusters, which we discuss below) to infer a lower supercluster mass estimate of $2.25 \times 10^{15}$ $M_{\odot}$. The total mass of 11 clusters in the UMa neighborhood (without A1279) is equal to $1.70 \times 10^{15}$ $M_{\odot}$. Some clusters of galaxies (A1270, RXCJ1010, and RXJ1033) contain subclusters located both within $R_{200}$ and near this radius, and therefore the inferred masses are lower estimates. Table 1 lists the derived parameters with the errors corresponding to the errors of inferred $\sigma$. The cluster centers usually coincide with the positions of the brightest galaxies of the corresponding clusters and are located close the centers of X-ray radiation (if detected) with the exception for some clusters discussed below. Table 1 also lists the $0.1-2.4 \mathrm{keV}$ X-ray luminosities adopted from the BAX database [8]. The number of galaxies, $N_{z}$, is equal to the number of galaxies observed within $R_{200}$ from the cluster center.

In figs 1-22 we present the following properties of the clusters: the deviations of the radial velocities of galaxies from the average radial velocity of the cluster within $\pm 3000 \mathrm{~km} / \mathrm{s}$; the integrated distribution of the number of galaxies as a function of the squared angular distance from the center of the cluster; the sky positions of galaxies within $45^{\prime}$ from the center of the cluster, and the distribution of the radial velocities of cluster member galaxies located within $R_{200}$ from the cluster center.

We compare our radial-velocity dispersions with those reported by Aguerri et al. [9] for the nine clusters in common to find that the differences are lying in the interval $(-53 \mathrm{~km} / \mathrm{s})<$ $\Delta \sigma<(+55 \mathrm{~km} / \mathrm{s})$. The differences for the A1291B, RXCJ1053A, and A1169 clusters are much greater and amount to $(+170 \mathrm{~km} / \mathrm{s}),(+158 \mathrm{~km} / \mathrm{s})$, and $(-149 \mathrm{~km} / \mathrm{s})$, respectively; they must be due to the presence of subclusters in these systems and to the differences in the techniques used to find the position of the cluster center and to identify cluster members. 
Table 1. Dynamic properties of clusters

\begin{tabular}{|c|c|c|c|c|c|c|c|}
\hline Cluster & $\begin{array}{c}\alpha(\mathrm{J} 2000) \delta \\
\mathrm{hh} \mathrm{mm} \text { ss dd } \mathrm{mm} \text { ss }\end{array}$ & $\begin{array}{c}c z_{h} \\
\mathrm{~km} / \mathrm{s}\end{array}$ & $N_{z}$ & $\begin{array}{c}\sigma_{c} \\
\mathrm{~km} / \mathrm{s} \\
\end{array}$ & $\begin{array}{l}R_{200} \\
\mathrm{Mpc}\end{array}$ & $\begin{array}{c}M_{200} \\
10^{14} M_{\odot} \\
\end{array}$ & $\begin{array}{r}L_{0.1-2.4 \mathrm{keV}} \\
10^{44} \mathrm{erg} / \mathrm{s}\end{array}$ \\
\hline $\mathrm{A} 1270$ & $112942.0+540556$ & 20688 & 57 & $553 \pm 73$ & 1.32 & $2.82 \pm 1.06$ & $0.06:$ \\
\hline A1291A & $113221.1+555803$ & 15394 & 33 & $391 \pm 68$ & 0.94 & $1.01 \pm 0.54$ & 0.22 \\
\hline A1291B & 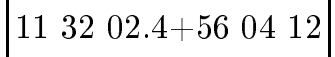 & 17357 & 37 & $550 \pm 90$ & 1.32 & $2.80 \pm 1.39$ & - \\
\hline A1318 & $1136 \quad 03.5+550431$ & 16914 & 40 & $394 \pm 62$ & 0.95 & $1.03 \pm 0.49$ & $0.04:$ \\
\hline A1377 & $114721.3+554349$ & 15531 & 86 & $632 \pm 68$ & 1.53 & $4.28 \pm 1.38$ & 0.28 \\
\hline A 1383 & $114805.8+543847$ & 17862 & 52 & $464 \pm 64$ & 1.12 & $1.69 \pm 0.74$ & $0.13:$ \\
\hline A1436 & $1200 \quad 08.8+56 \quad 1052$ & 19499 & 89 & $682 \pm 72$ & 1.64 & $5.34 \pm 1.70$ & 0.52 \\
\hline Anon1 & $111523.8+542639$ & 20951 & 55 & $608 \pm 82$ & 1.46 & $3.78 \pm 1.53$ & 0.35 \\
\hline Anon2 & $111946.0+542802$ & 21147 & 14 & $253 \pm 68$ & 0.61 & $0.27 \pm 0.22$ & - \\
\hline Anon3 & $112932.3+552520$ & 20390 & 23 & $375 \pm 78$ & 0.90 & $0.88 \pm 0.56$ & - \\
\hline Anon 4 & $113908.5+553952$ & 18303 & 25 & $397 \pm 79$ & 0.95 & $1.05 \pm 0.64$ & - \\
\hline Sh166 & $1203 \quad 11.9+545050$ & 15003 & 24 & $327 \pm 67$ & 0.79 & $0.59 \pm 0.38$ & - \\
\hline A1003 & $102501.6+475028$ & 18882 & 28 & $562 \pm 106$ & 1.35 & $2.98 \pm 1.95$ & 0.10 \\
\hline A1169 & $1107 \quad 49.3+435500$ & 17630 & 69 & $582 \pm 70$ & 1.40 & $3.32 \pm 1.20$ & 0.06: \\
\hline A 1279 & $113139.3+67 \quad 1430$ & 16285 & 6 & $187 \pm 76$ & 0.45 & $0.11 \pm 0.14$ & - \\
\hline A 1452 & $120328.4+514256$ & 18542 & 20 & $514 \pm 115$ & 1.23 & $2.27 \pm 1.71$ & - \\
\hline A1461 & $120424.7+423343$ & 16177 & 13 & $317 \pm 88$ & 0.77 & $0.54 \pm 0.46$ & - \\
\hline A 1507 & $121448.6+595422$ & 17978 & 38 & $432 \pm 70$ & 1.04 & $1.36 \pm 0.66$ & 0.07 \\
\hline A1534 & $122442.8+612815$ & 20967 & 18 & $307 \pm 72$ & 0.74 & $0.49 \pm 0.35$ & - \\
\hline RXCJ1010 & $101016.1+543006$ & 13736 & 34 & $418 \pm 72$ & 1.01 & $1.24 \pm 0.60$ & 0.02 \\
\hline RXJ1033 & $103351.2+570321$ & 13671 & 47 & $413 \pm 60$ & 1.00 & $1.19 \pm 0.52$ & 0.01 \\
\hline RXCJ1053A & $1054 \quad 11.2+545018$ & 21551 & 49 & $507 \pm 72$ & 1.21 & $2.18 \pm 0.94$ & 0.53 \\
\hline RXCJ1053B & $105147.0+552300$ & 22113 & 30 & $420 \pm 77$ & 1.00 & $1.23 \pm 0.42$ & - \\
\hline RXCJ1122 & $1122 \quad 45.8+670955$ & 16607 & 12 & $223 \pm 64$ & 0.54 & $0.19 \pm 0.16$ & 0.06 \\
\hline
\end{tabular}

\subsection{Comments on Some Clusters}

A1270. This cluster is located in the farthermost filament of the UMa system (see [5, Fig. 1c]). It is evident from Fig. 1 that the cluster has emerged from the filament, however, there are still subsystems within $R_{200}$. The cluster does not have the brightest central galaxy and we adopt the midpoint between the the two main subsystems as the position of the cluster center.

A1291A,B. The A1291A cluster (Fig. 2) has a dominating galaxy. We adopted its position as the position of the cluster center, although the center of X-ray radiation is slightly offset with respect 

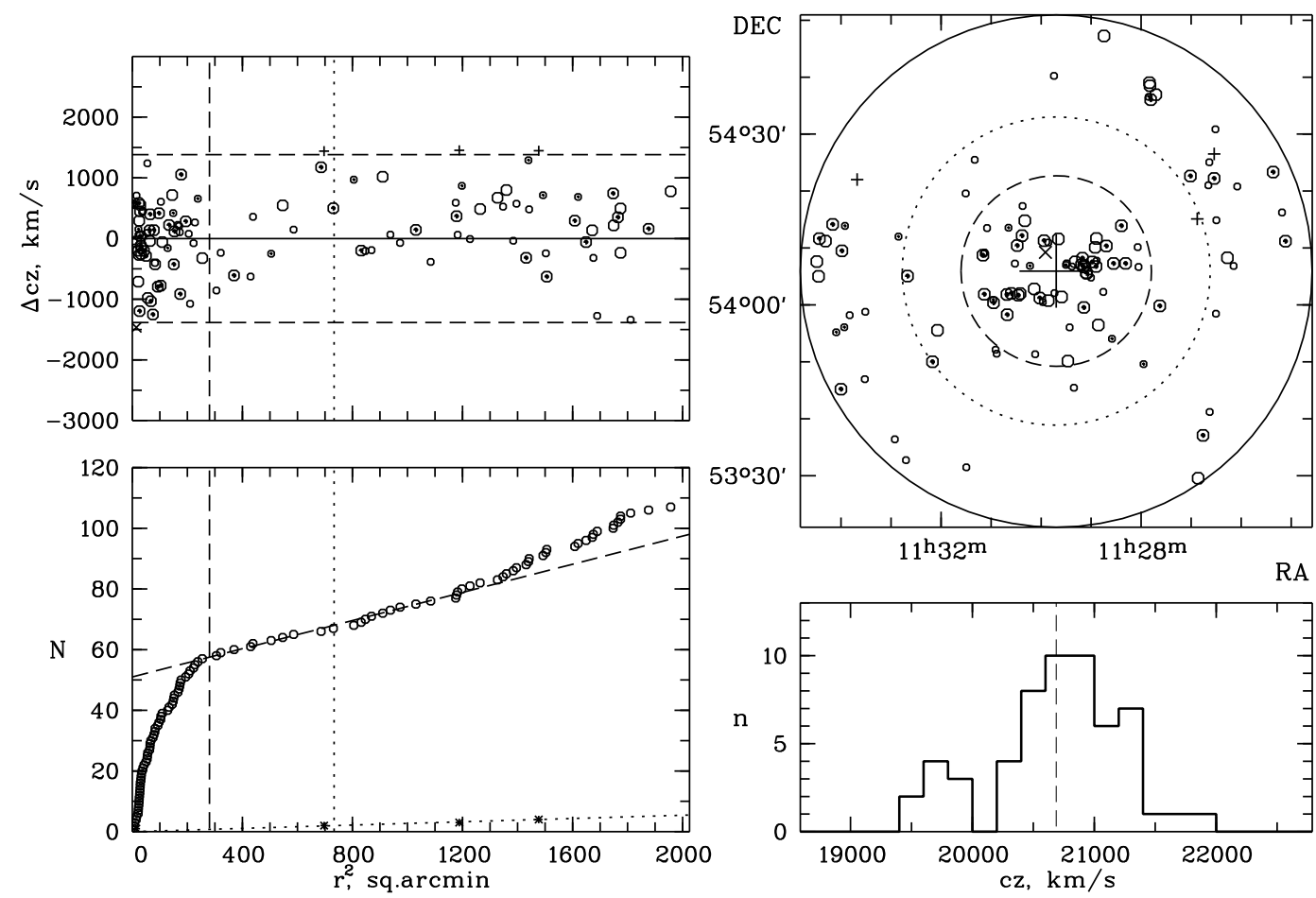

Figure 1. Distribution of galaxies in A1270. The top left figure represents the deviation of the radial velocities of galaxies from the mean radial velocity of the cluster, averaged over the galaxies located within $R_{200}$ from the cluster center. The horizontal dashed lines correspond to $\pm 2.5 \sigma$ deviations; the vertical dashed line indicates the $R_{200}$ radius, and the dotted line shows the Abell radius $(2.14 \mathrm{Mpc})$. The large circles indicate the galaxies brighter than $M_{K}^{*}+1=-23^{m} .29$; the circles with dots inside are the early-type galaxies; plus signs and crosses are the background and foreground galaxies, respectively. In the bottom left figure we present the integrated distribution of the total number of galaxies as a function of the squared clustercentric distance. The circles and asterisks correspond to the galaxies shown by circles in the top left figure and to background galaxies, respectively. The dashed and dotted lines indicate the domains of linear increase of the number of cluster and background galaxies, respectively. In the top right figure you can see the sky distribution (in equatorial coordinates) of the galaxies represented in the top left figure (the same designations are used). Centered circles denote the regions inside $R_{200}$ (the dashed curve) and the Abell radius (the dotted curve). The domain under study is bounded by the circle of radius $45^{\prime}$ (the solid line). The big cross indicates the position of the center of the cluster. And finally in the bottom right figure we presented the distribution of the radial velocities of the cluster galaxies located within $R_{200}$. The vertical dashed line corresponds to the average radial velocity of the cluster. The structure and designations are the same in figs. $1-22$.

to it. The A1291B cluster deviates significantly from the relations derived below (see Section 5), and at least two subsystems are apparent in its radial-velocity distribution (Fig. 3) (the bottom right figure). A plausible explanation would be to assume that the A1291B cluster is actually a projection of several groups of galaxies oriented along the line of sigh. In this case the dispersion 

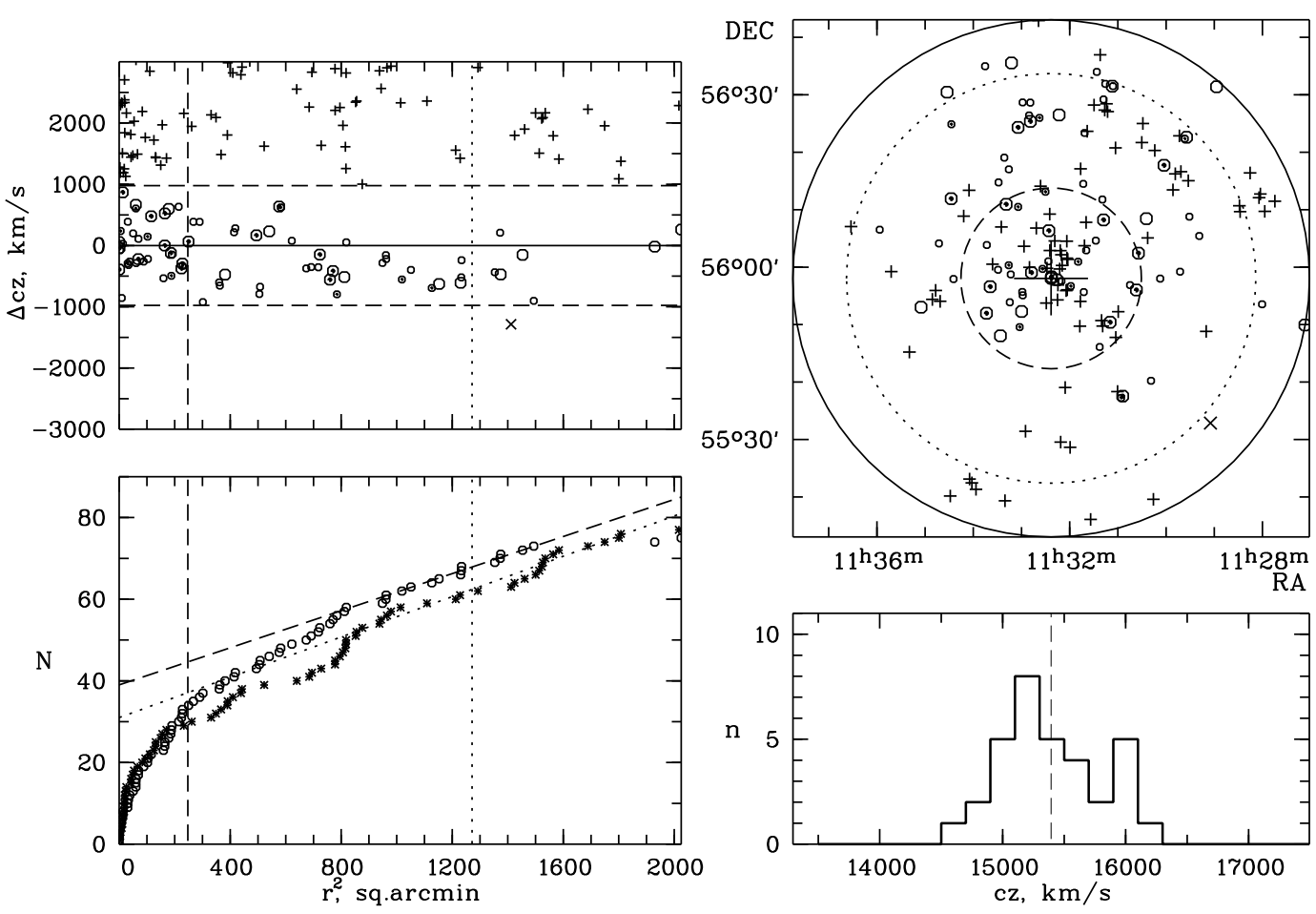

Figure 2. Distribution of galaxies in A1291A.
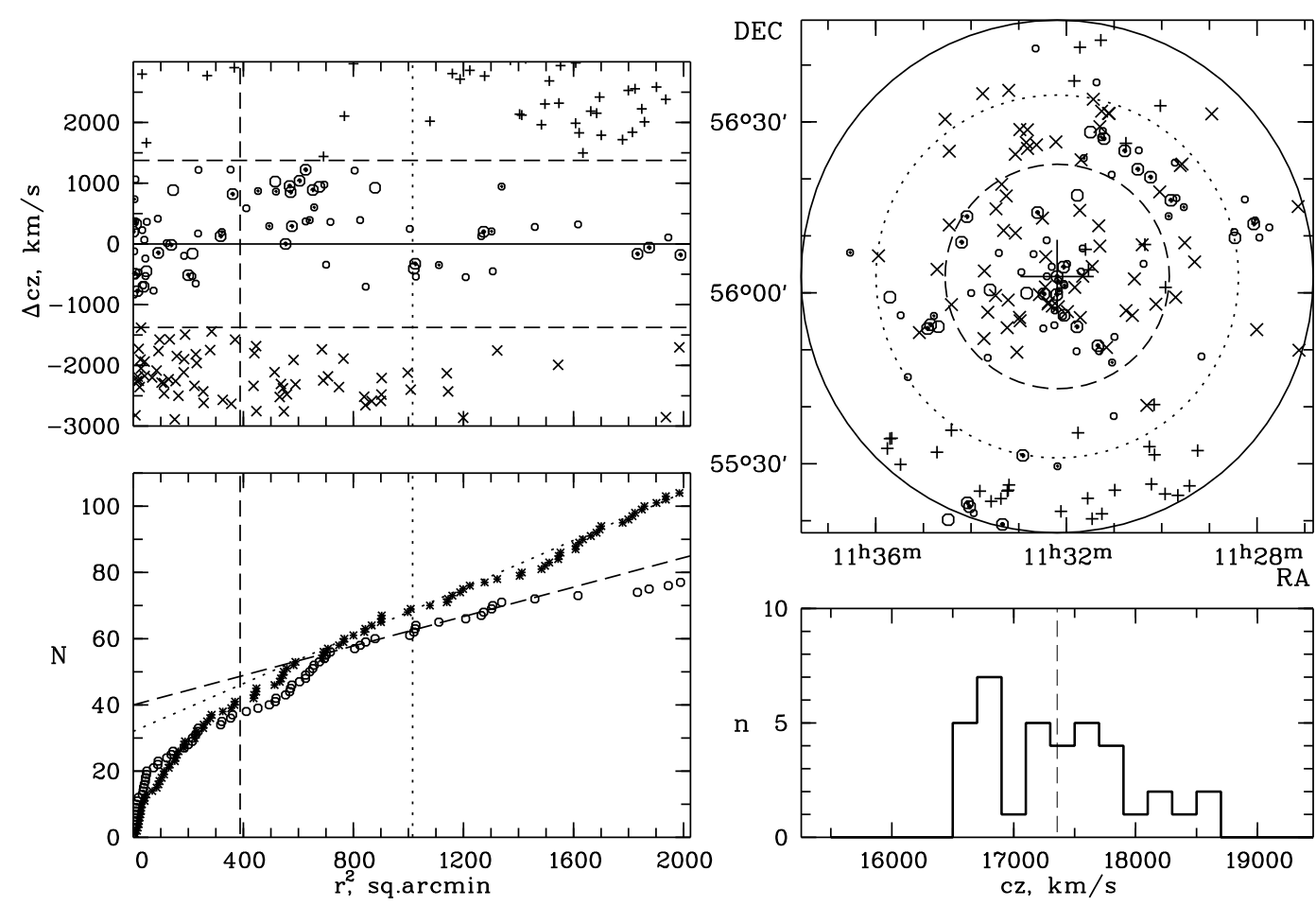

Figure 3. Distribution of galaxies in A1291B. 

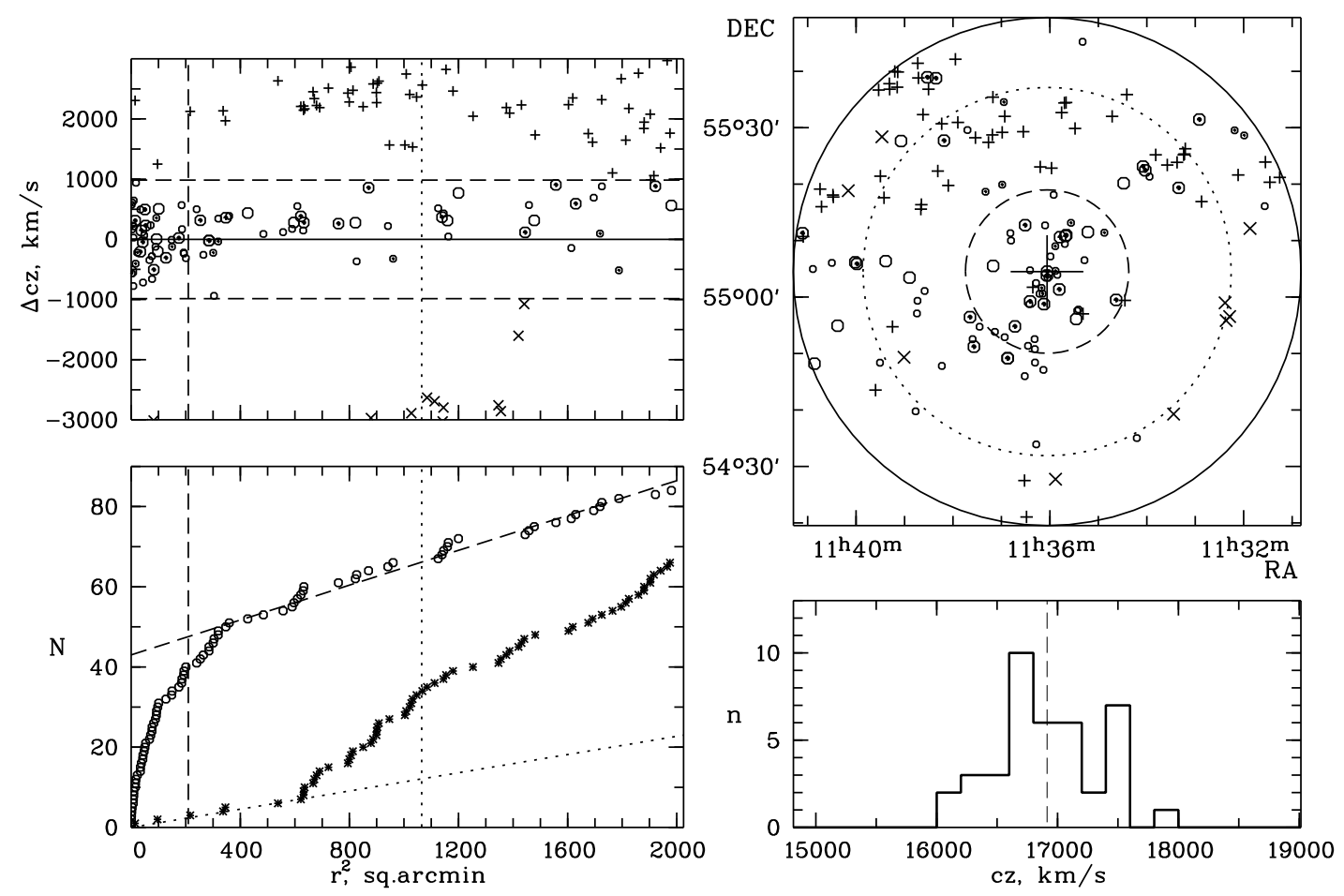

Figure 4. Distribution of galaxies in A1318.
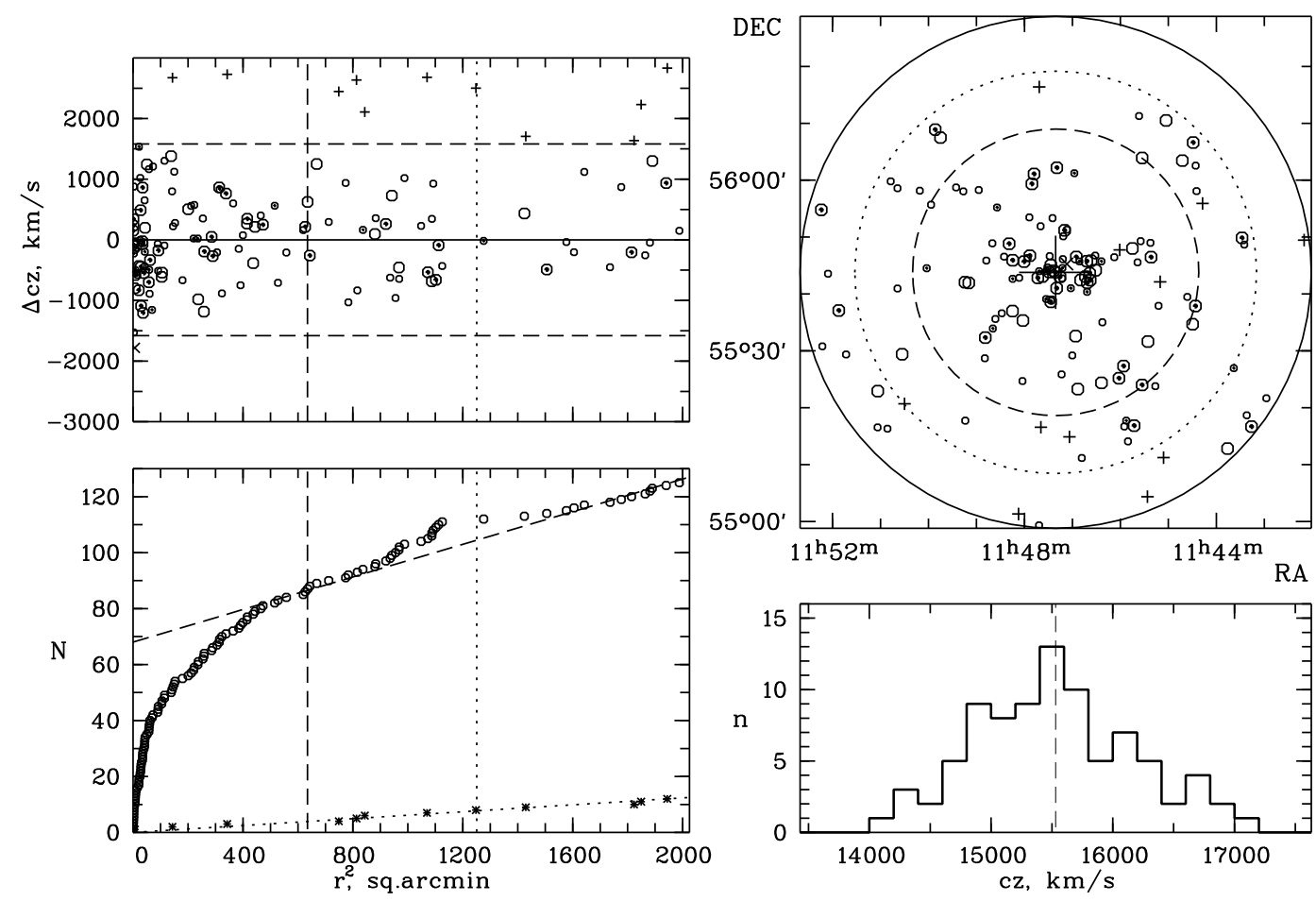

Figure 5. Distribution of galaxies in A1377. 

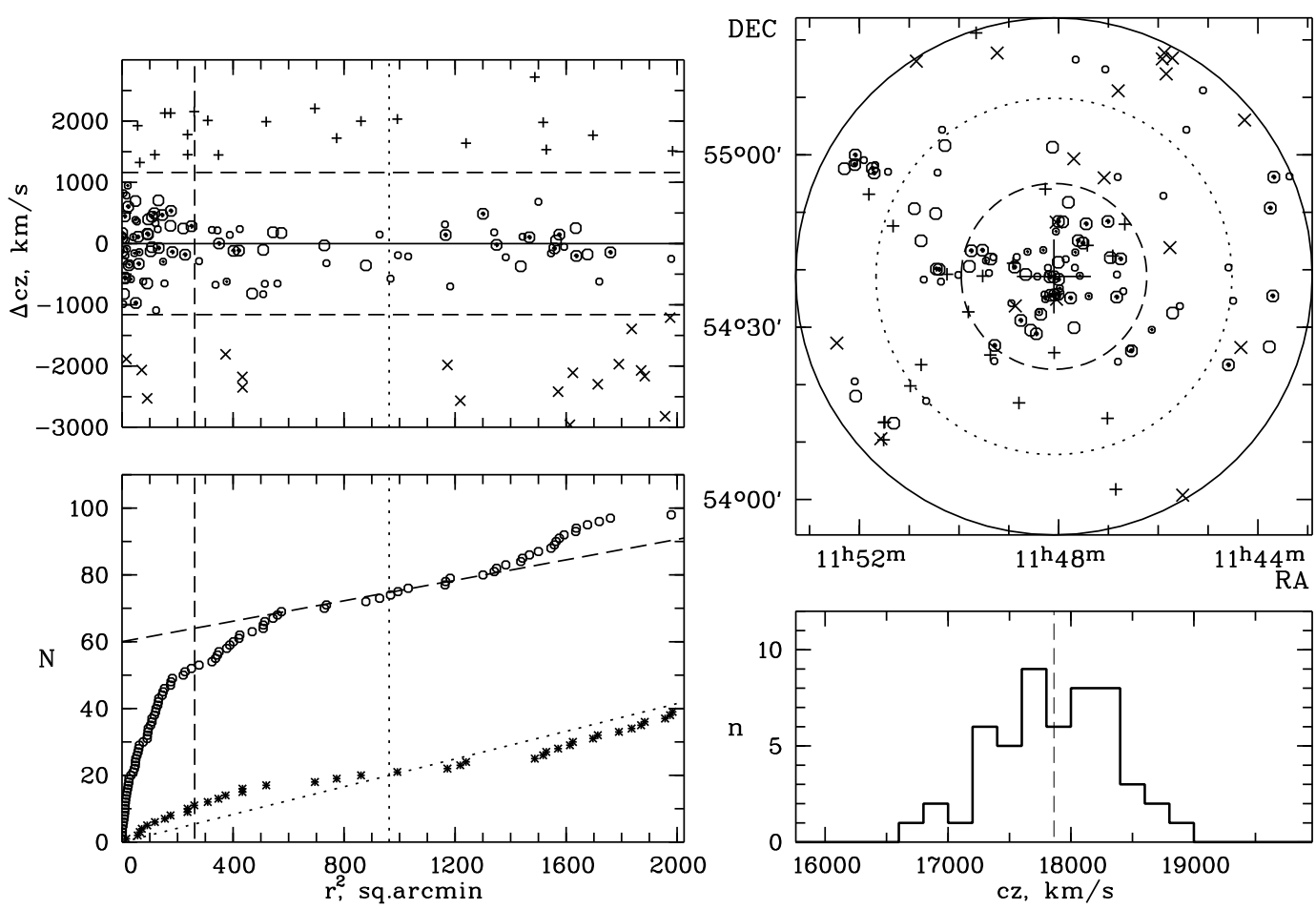

Figure 6. Distribution of galaxies in A1383.
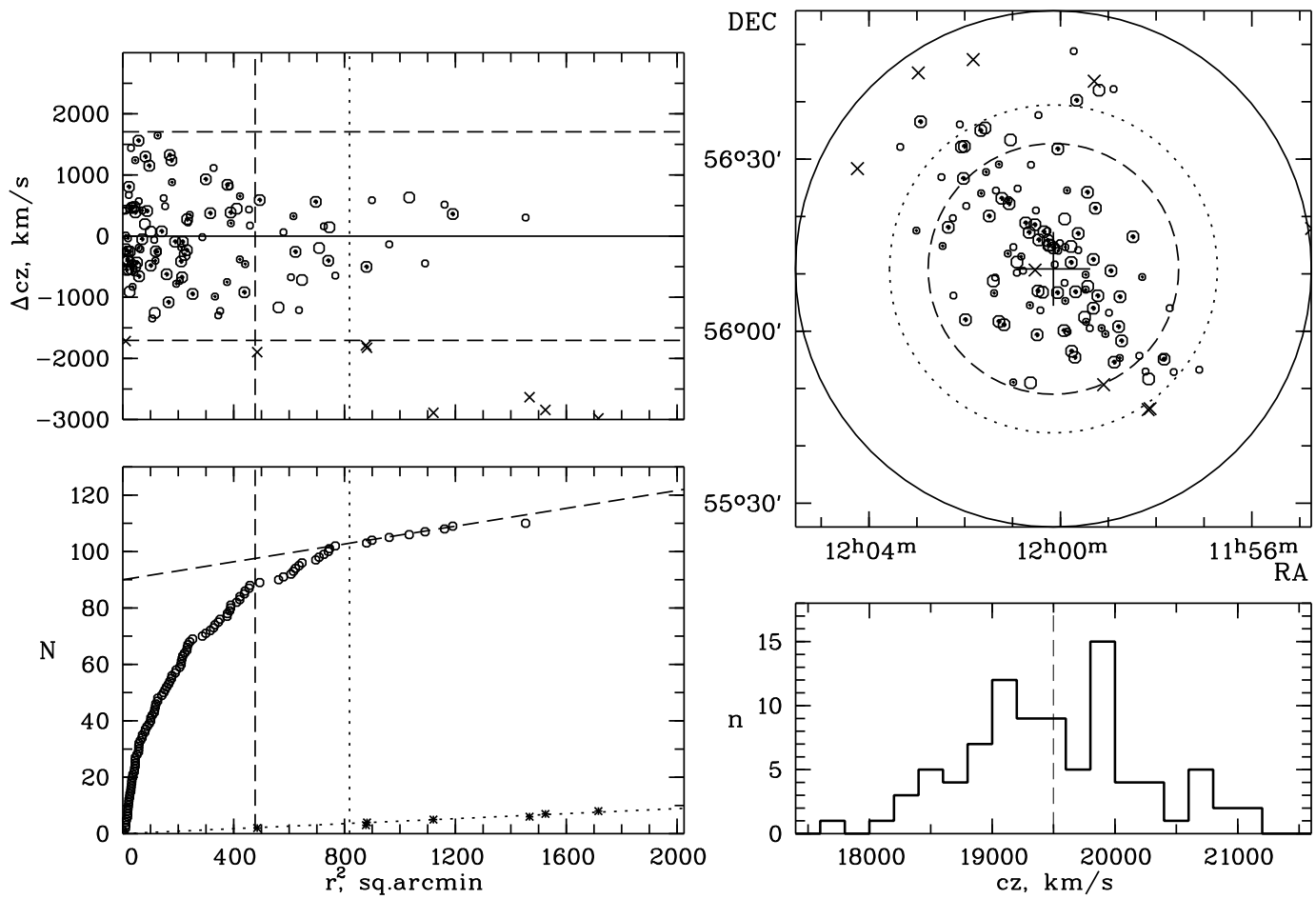

Figure 7. Distribution of galaxies in A1436. 
of galaxy velocities and the mass of the cluster should be highly overestimated.

A1436. The cluster has an elongated shape and sharp edges. We may be observing it during a period of violent dynamical relaxation after the merger of two subclusters along the filament, when most of the galaxies have already concentrated inside the virial radius, but the brightest galaxy had not yet settled at the center of the cluster. As the center of the cluster we adopted the position given by the mean coordinates of the two brightest galaxies.

Anon1 (RXCJ1115.5+5426) and Anon2. These clusters are located in the farthermost filament of the UMa supercluster (see [5, Fig. 1c]). Anon1 (Fig. 8) is a rather rich X-ray cluster with a cD galaxy at its center, which may be a part of the filament adjacent to the Anon1 cluster rather than an isolated virialized object. The Anon2 cluster, which is located 30' East of Anon1, is a group consisting of bright early-type galaxies with a low dispersion of radial velocities. That is why Anon2 does not obey the relations that we derive for normal clusters (Section 5).

A1169. The cluster (Fig. 13) is highly elongated in the sky plane from Northeast to Southwest. As its center, we adopted the centroid computed over all galaxies, although the brightest galaxy is located in the Southwestern compact subgroup of galaxies.

A1279. A very poor cluster (Fig. 14) with one elliptical galaxy and several late-type galaxies located within $R_{200}$. This cluster has the lowest fraction $(0.25)$ of early-type galaxies down to a limiting magnitude of $M_{K}^{*}+1$. By its parameters, A1279 should be classified as a group of galaxies.

RXCJ1010. This cluster has not yet entirely formed (Fig. 19). The center of the cluster is located at the center of X-ray flux distribution, which coincides with the position of the brightest galaxy. It is evident from Fig. 19 (the top right figure) that the cluster contains small subclusters located near the center (about $9^{\prime}$ and $18^{\prime}$ from it), which consist of early-type galaxies.

RXJ1033. The coordinates of the center of the X-ray flux distribution approximately coincide with those of the brightest galaxy. However, given the presence of another subcluster located $15^{\prime}$ from this center (Fig. 20), which consists mostly of late-type galaxies and has a radial velocity that differs by $300 \mathrm{~km} / \mathrm{s}$ from that of the main cluster, we assume that the center of the cluster should coincide with the position of the centroid computed over all galaxies of the cluster.

RXCJ1053A,B. There is another cluster (which we designate as RXCJ1053B) 33' Northwest of the main cluster discovered via X-ray observations (Fig. 21). The average radial velocity of RXCJ1053B differs by about $600 \mathrm{~km} / \mathrm{s}$ from that of the main cluster.

RXCJ1122. We set the center of the cluster (Fig. 22) at the midpoint between the two brightest galaxies. 

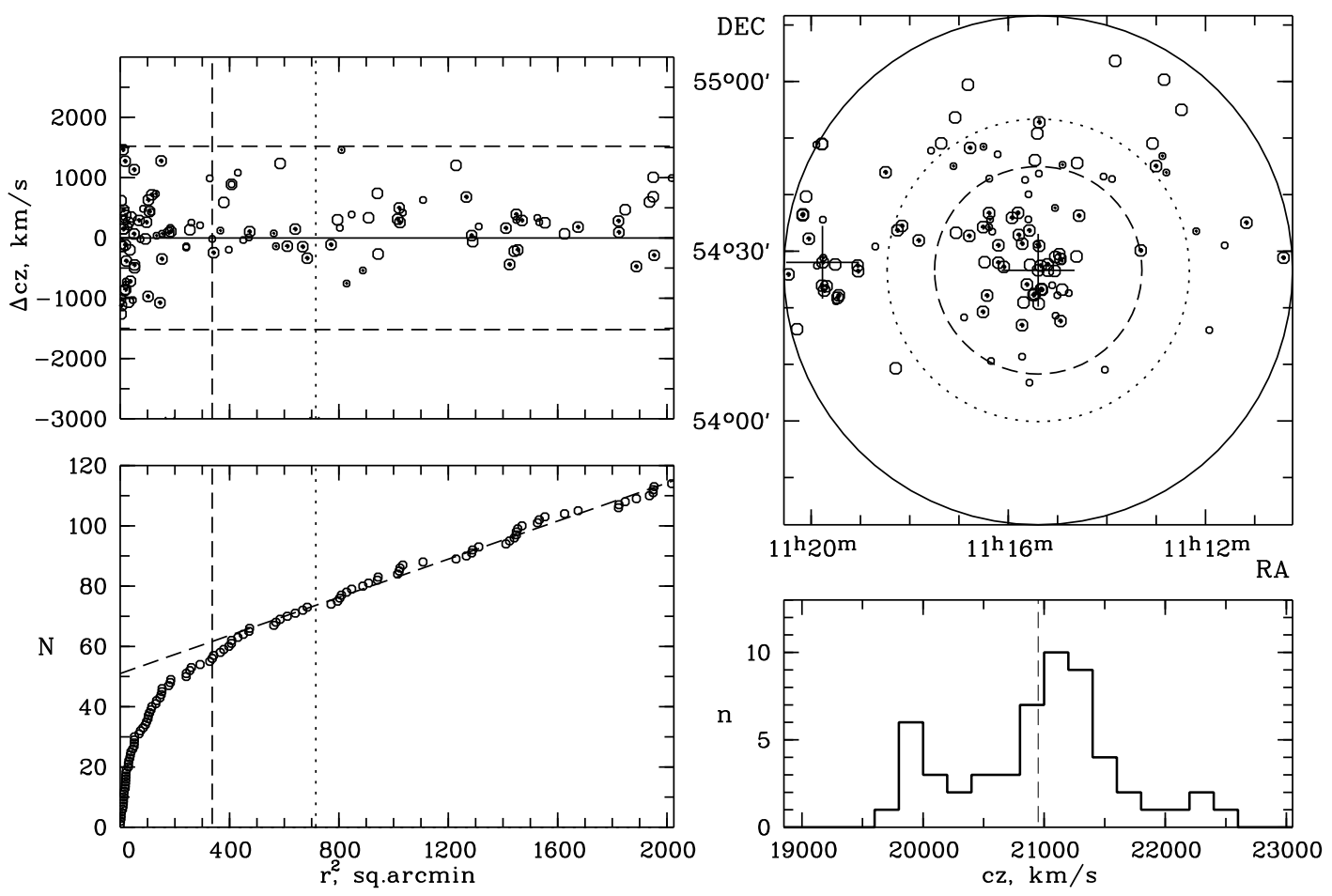

Figure 8. Distribution of galaxies in the Anon1 cluster (the Anon2 cluster is located left of Anon1 and we indicate the position of its center by a big cross).
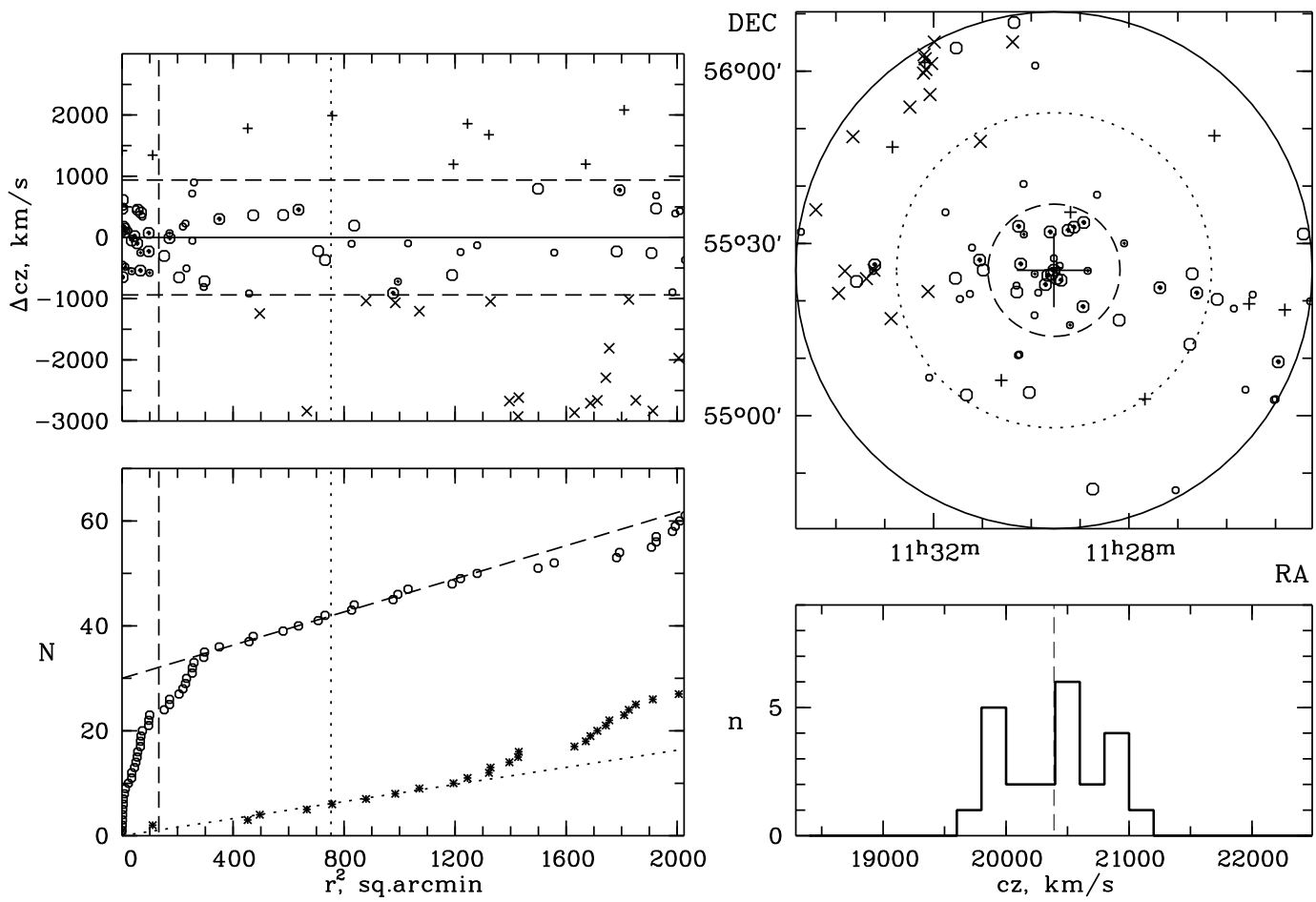

Figure 9. Distribution of galaxies in Anon3. 

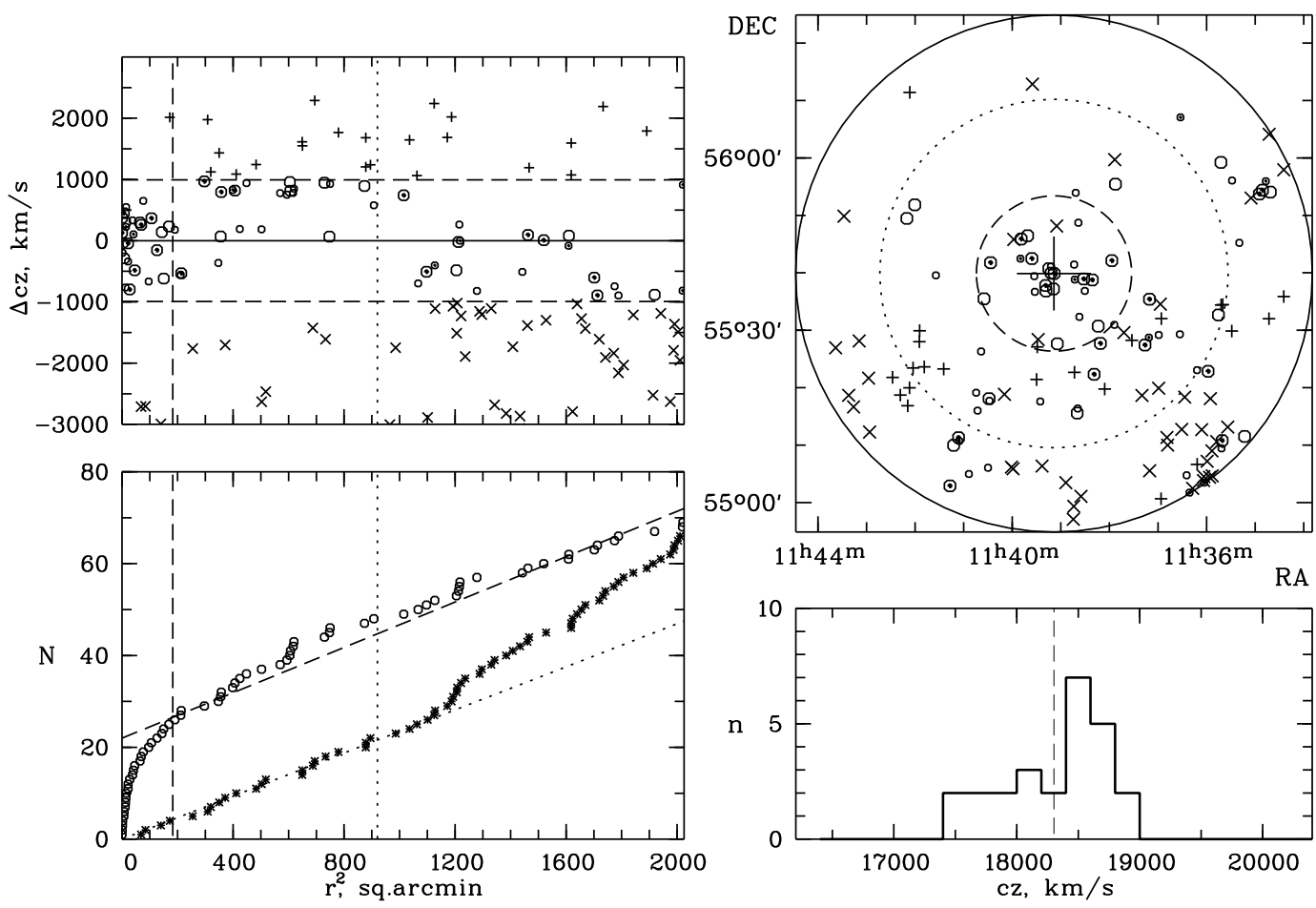

Figure 10. Distribution of galaxies in Anon4.
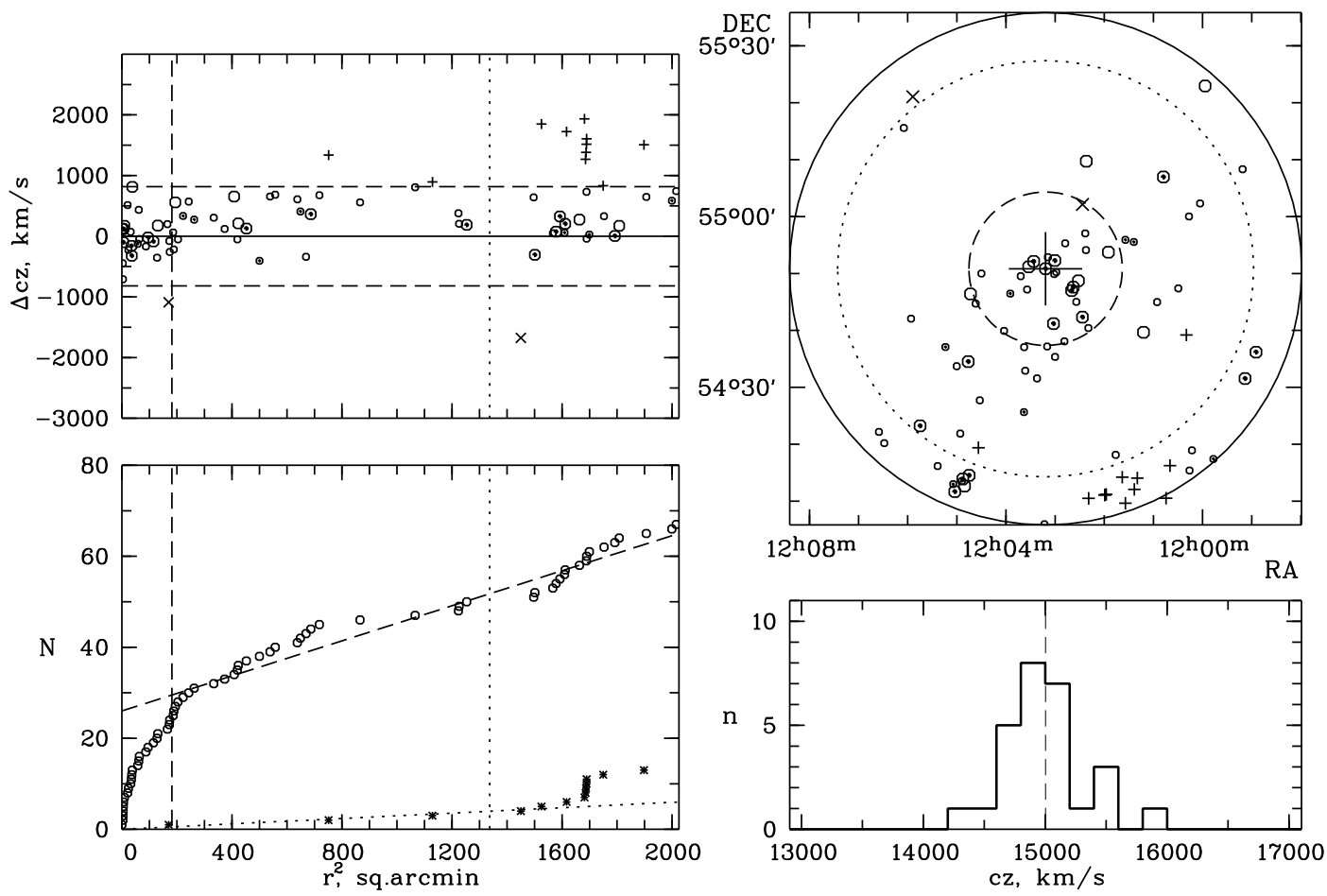

Figure 11. Distribution of galaxies in Sh166. 


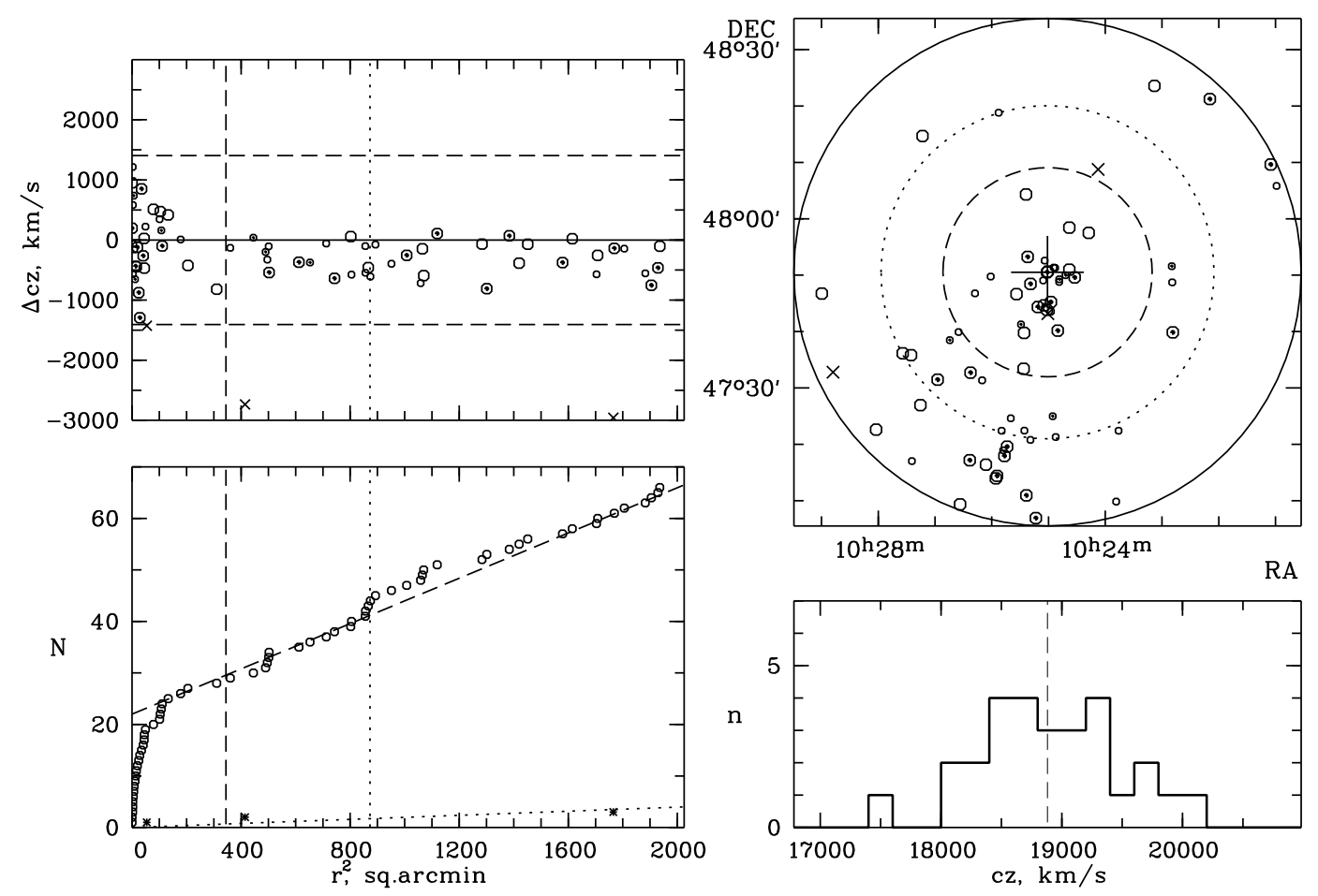

Figure 12. Distribution of galaxies in A1003.
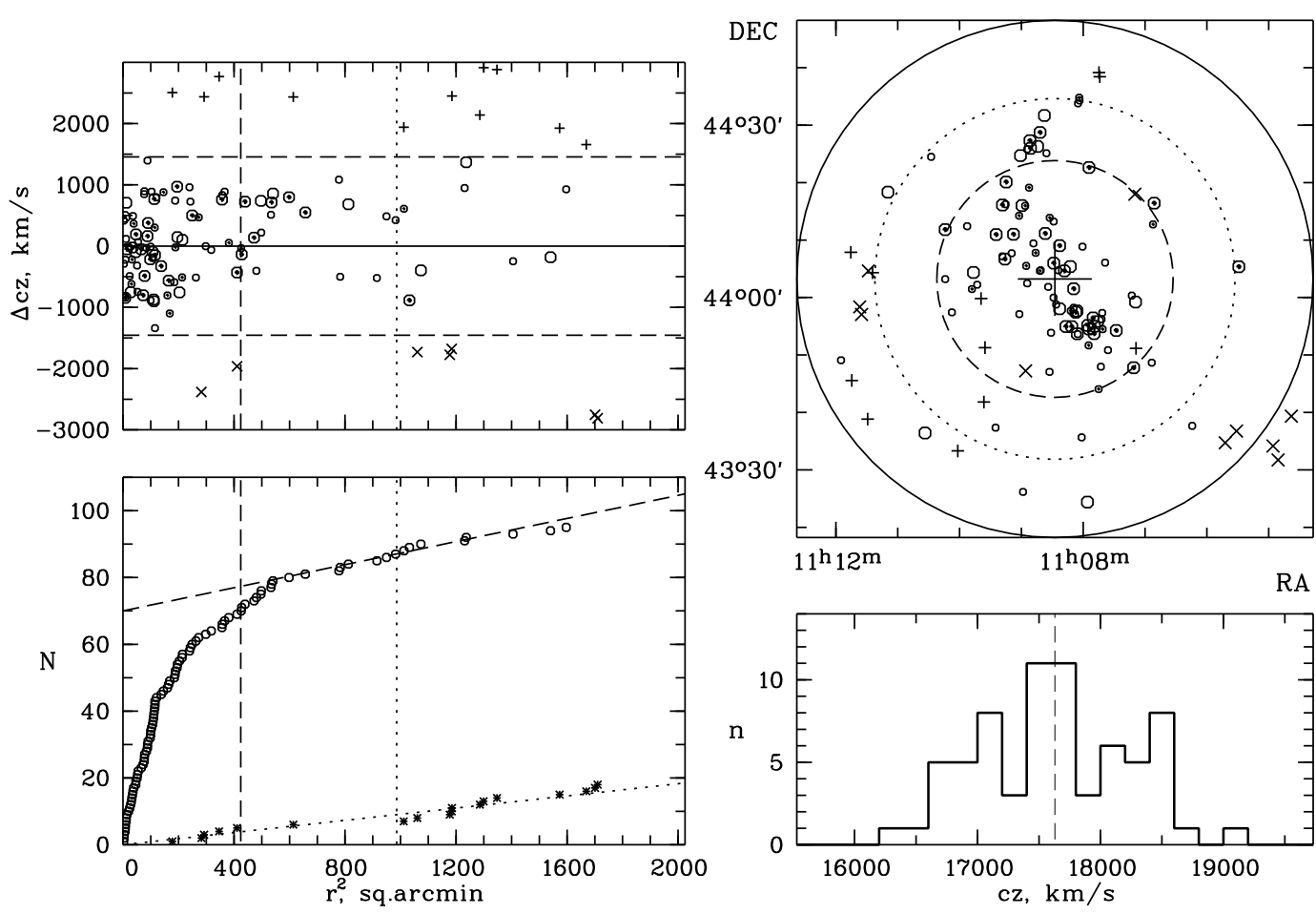

Figure 13. Distribution of galaxies in A1169. 

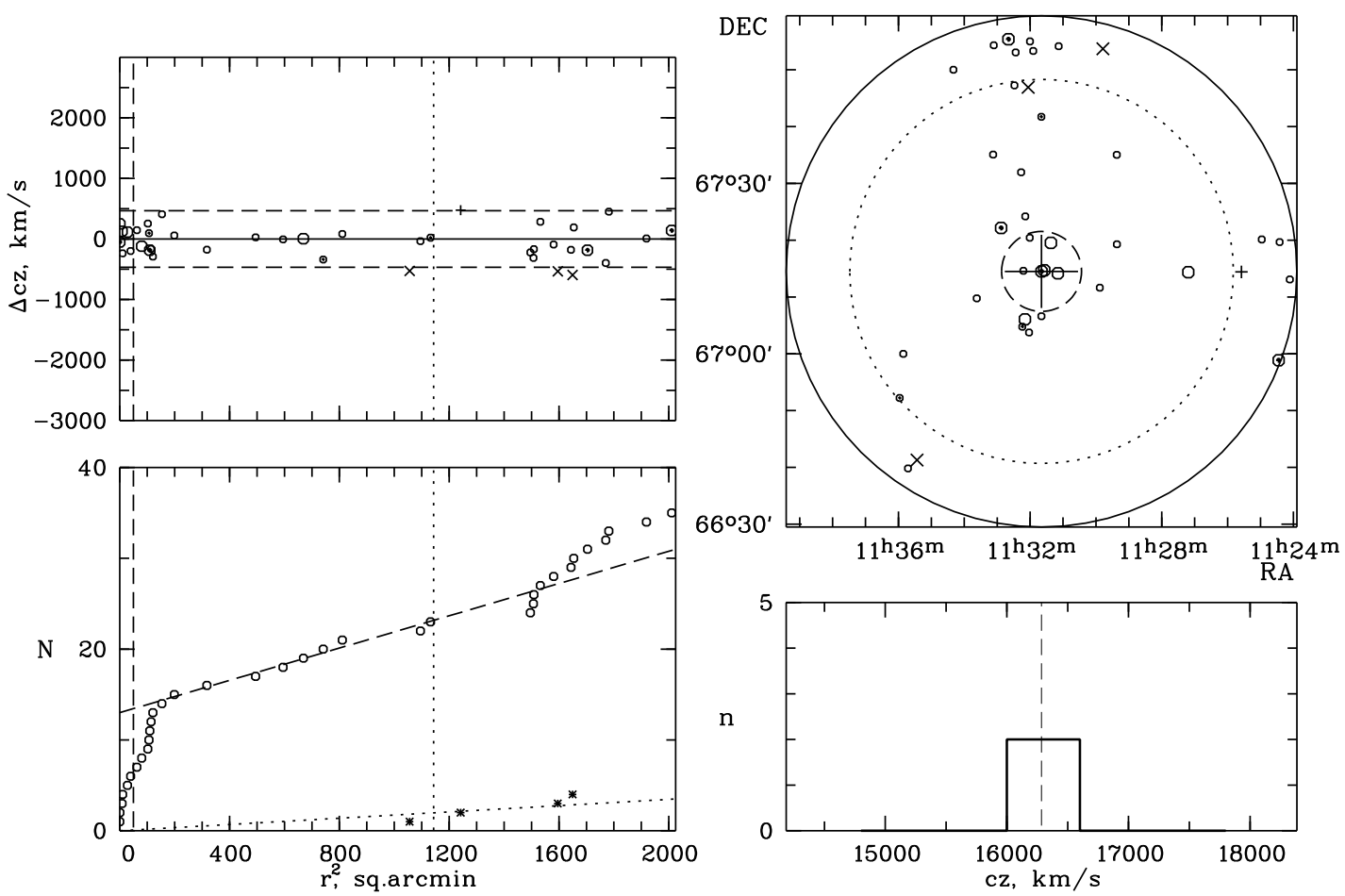

Figure 14. Distribution of galaxies in A1279.
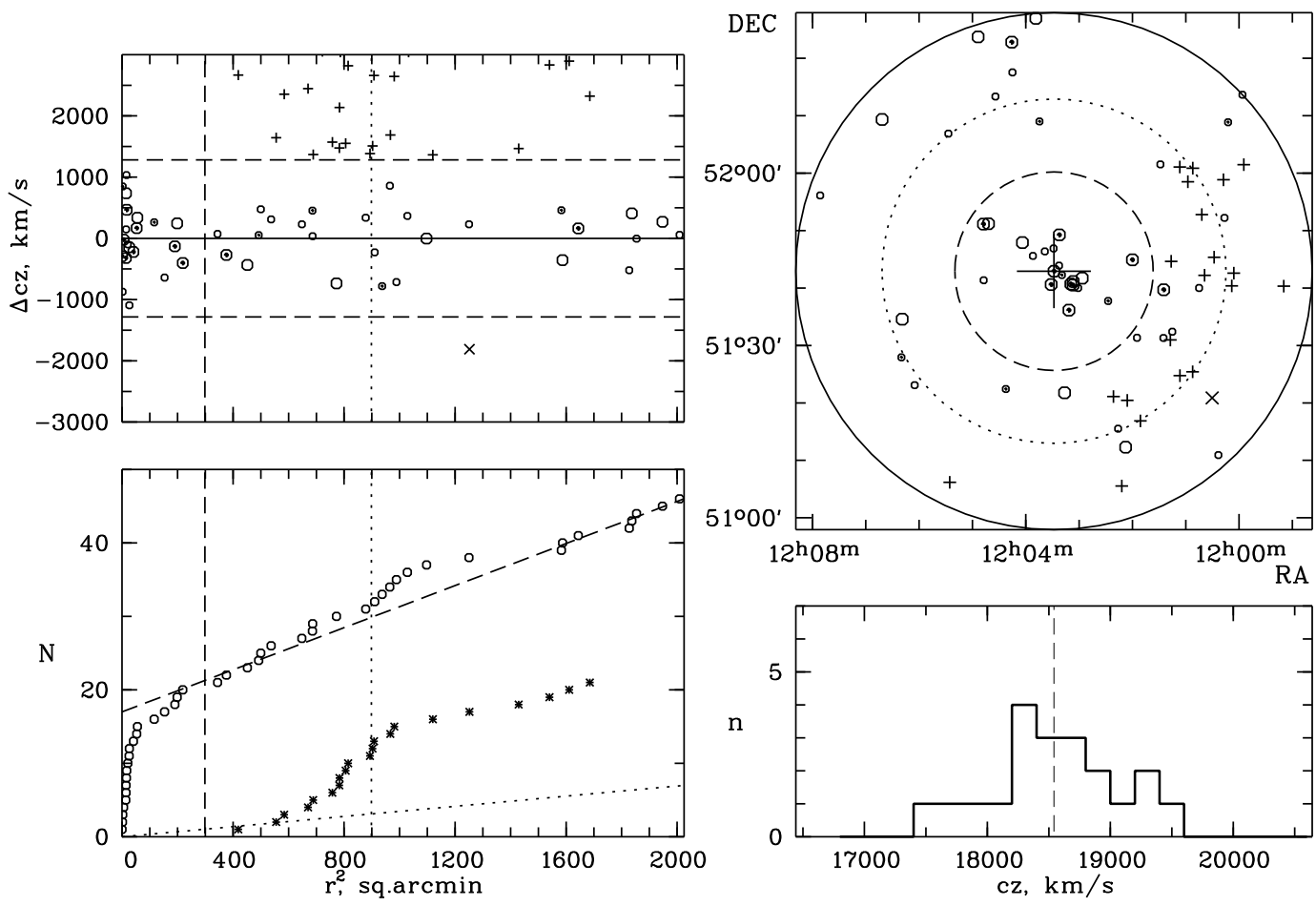

Figure 15. Distribution of galaxies in A1452. 


\section{TOTAL $K$-BAND LUMINOSITY OF CLUSTERS OF GALAXIES}

The main baryon components of clusters of galaxies are: stars in galaxies and in the intergalactic space and the hot gas that fills the intergalactic and intercluster space. The parameters of these baryonic components can be measured in the process of observations. Infrared (IR) radiation of stars is not affected significantly by either starbursts in a galaxy or dust, because the central regions of clusters of galaxies are mostly occupied by early-type galaxies with old stellar population. IR radiation is therefore a more accurate tracer of mass of the stellar population in clusters of galaxies and it is often used for this (see, e.g., [10-12]). To find the total IR luminosities of clusters of galaxies, in our earlier paper [6] we used the photometric data given in the final release of the 2MASS catalog for extended objects (XSC [13]). About a half of the galaxies (at the distance of the UMa system $(z \simeq 0.06)$ ) discovered spectroscopically in the SDSS catalog have no measurements in the XSC and we therefore used the data from the point-source catalog (PSC) for these objects. The magnitudes of bright galaxies, listed in this catalog, have rather large errors, although the magnitude corrections for about $14^{m}$ galaxies (which are usually absent in the XSC catalog) are about $0.2^{m}$. Figure 23 demonstrates, as an example, the $K(X S C)-K(P S C)$ differences for the same galaxies of the A1377 cluster.

In this paper to search for the $K$-band magnitudes we use a different method described by M. Obrić et al. [14]. They calculate the $K(S D S S)$ magnitudes for 99000 SDSS (DR1) galaxies based on their $u-r$ color indices. We proceeded as follows: $K(S D S S)=r_{p e t}-(r-$ $K)$, where $r_{p e t}$ is the Petrosian $r$-band magnitude of the galaxy and $r-K$ is given by the formula $r-K=1.115+0.94(u-r)-0.165(u-r)^{2}+0.00851(u-r)^{3}+4.92 z-9.1 z^{2}$ (here $z$ is the redshift of the galaxy). We further corrected the $r-K$ colors by adding the terms $0.496-0.154 R_{50}^{z}$ for late-type $(u-r<2.22)$ and $0.107-0.045 R_{50}^{z}$ for early-type $(u-r \geq 2.22)$ galaxies, respectively, where $R_{50}^{z}$ is the radius of the region emitting $50 \%$ of the Petrosian $z$-band flux. We adjusted Petrosian's magnitudes as described by Graham [15] to transform them into the total galaxy magnitudes by the formula:

$$
r_{\text {tot }}=r_{\text {pet }}-5.1 \times 10^{-4} \times \exp \left(\left(R_{90}^{r} / R_{50}^{r}\right)^{1.451}\right)
$$

where $R_{90}^{r}$ and $R_{50}^{r}$ are the radii of the regions containing $90 \%$ and $50 \%$ of the Petrosian $r$-band flux, respectively. Figure 24 represents the difference between the computed $(K(S D S S))$ and integrated $(K(X S C))$ magnitudes obtained from isophotal magnitudes corresponding to the surface brightness level of $\mu_{K}=20^{m} / \square^{\prime \prime}$, minus $0.2^{m}$ in accordance with the recommendations of Kochanek et al. [16]. E.g., the corresponding magnitude difference for 92 galaxies $\left(r_{p e t}<17.77^{m}\right)$ of the A1377 cluster is 

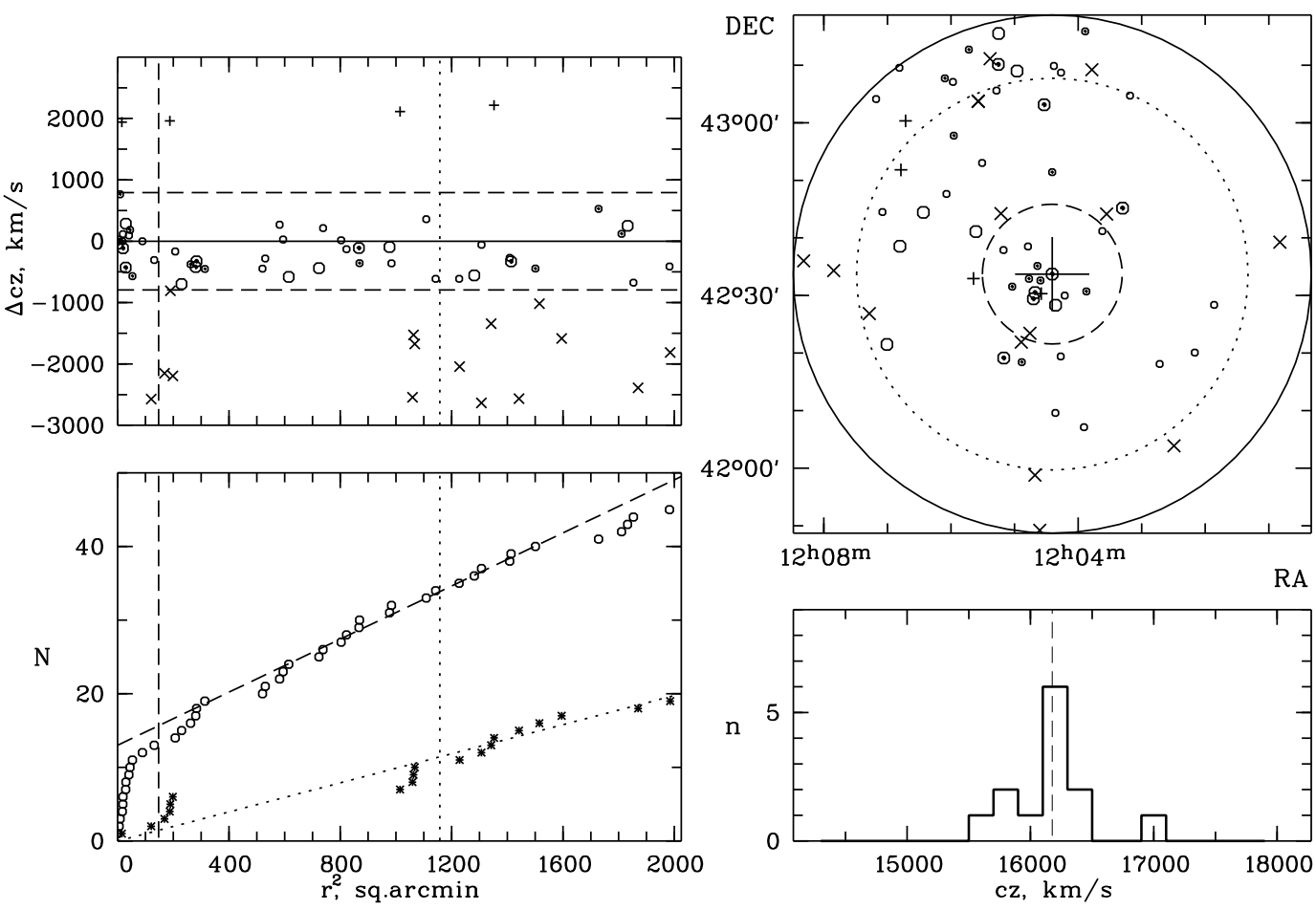

Figure 16. Distribution of galaxies in A1461.
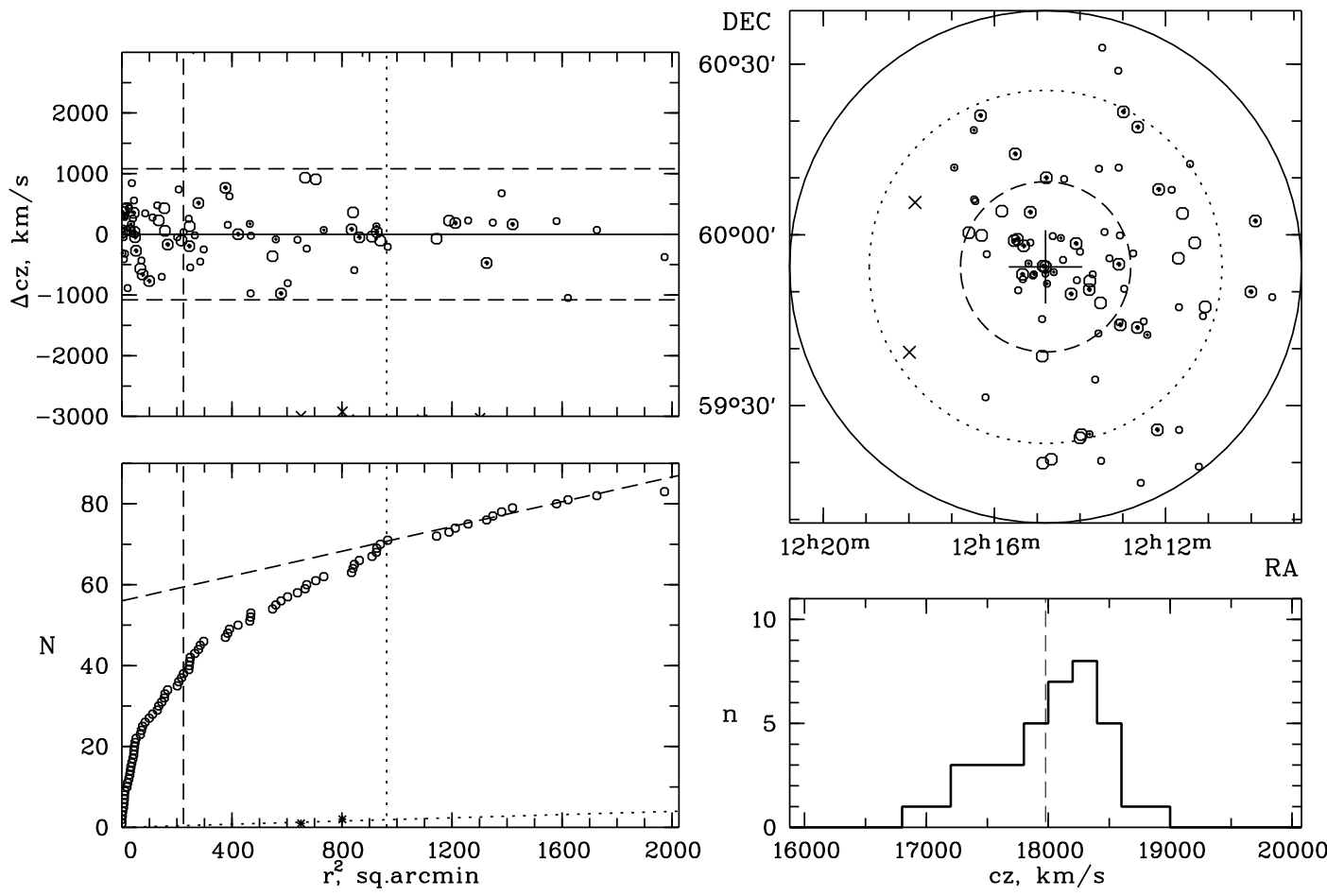

Figure 17. Distribution of galaxies in A1507. 
equal to $(0.12 \pm 0.02)^{m}$, and it is the same for other clusters within the quoted errors. We applied this correction when computing $K(S D S S)$. The average error of the isophotal magnitudes in the extended source catalog is equal to $0.1^{m}$ for the clusters studied.

The completeness of our sample is determined by that of the spectroscopic data of the SDSS catalog. For objects satisfying the conditions $r_{p e t}<17.77^{m}$ and $\mu_{r}<24.5^{m} / \square^{\prime \prime}$ (the Petrosian $r$-band magnitude of the galaxy corrected for Galactic absorption and the average Petrosian surface brightness corresponding to the effective radius) the completeness of SDSS data is estimated at $99 \%$ [17] and the completeness for bright galaxies, at 95\%. We supplemented the radial-velocity measurements for bright galaxies (one to five objects per cluster), which are unavailable in SDSS, by adopting them from the NED database.

The $L_{200, K}$ luminosity of a cluster is equal to the sum of the $K$-band luminosities of its member galaxies located within $R_{200}$ down to a fixed limiting absolute magnitude. We set this limit, like $\operatorname{Lin}$ et al. [10], equal to $-21^{m}$. We first transformed the observed galaxy magnitudes into the corresponding absolute magnitudes by the formula: $M_{K}=K-25-5 \log _{10}\left(D_{l} / 1 \mathrm{Mpc}\right)-A_{K}-K(z)$, where $D_{l}$ is the distance to the galaxy used to compute its luminosity; $A_{K}$ is the Galactic extinction and $K(z)=-6 \log (1+z)$, the K-correction computed in accordance with [16]. We applied no evolutionary corrections because of the small redshift interval (from 0.045 to 0.075 ) spanned by the objects in our sample. Galactic extinction, which we adopt from NED, is less than $0.01^{m}$ for our galaxies. The 2MASS (XSC) is not a deep survey (its $K$-band photometric limit for the completeness level of $90 \%$ and above is equal to $13.5^{m}$ in $K$-band [13]). We supplemented this catalog with galaxies from SDSS catalog with the Petrosian magnitudes $r_{\text {pet }}<17.77^{m}$. Given that the $r-K$ color index is, on the average, equal to $2.8^{m}$ for early-type galaxies, which make up most of galaxies within $R_{200}$, the limiting $K$-band magnitude of our sample of galaxies with individual $K$-band magnitude estimates must be of about $15^{m}$.

The computation of the total luminosity of a cluster of galaxies using the luminosity function (LF) inside the selected radius is a two-stage procedure [11]: the LF should first be normalized to the observed number of galaxies and then extrapolated into the domain of faint magnitudes down to the adopted limit. This is generally done either using the parameters of the Schechter function (the characteristic magnitude $M_{K}^{*}$ and the slope $\alpha$ ) for field galaxies or using the corresponding parameters determined for the composite LF of the sample studied. To estimate how the computed luminosities of clusters differ in these two cases, we first used the parameters of the Schechter function for field galaxies as inferred by Kochanek et al. [16] $\left(M_{K}^{*}, \alpha\right)=\left(-24.14^{m},-1.09\right)$, which are commonly employed by other authors. We computed the normalization parameter for each 

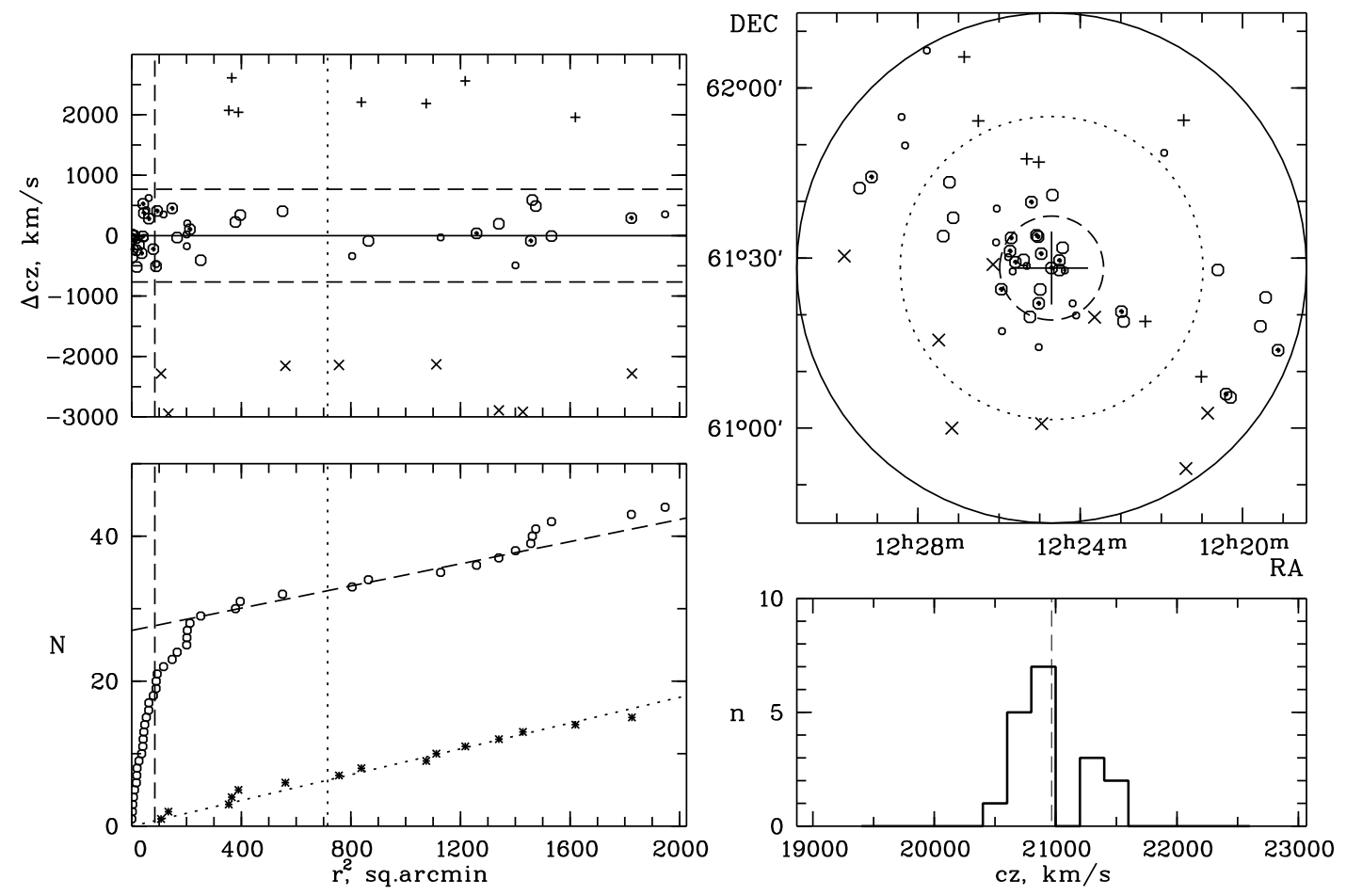

Figure 18. Distribution of galaxies in A1534.
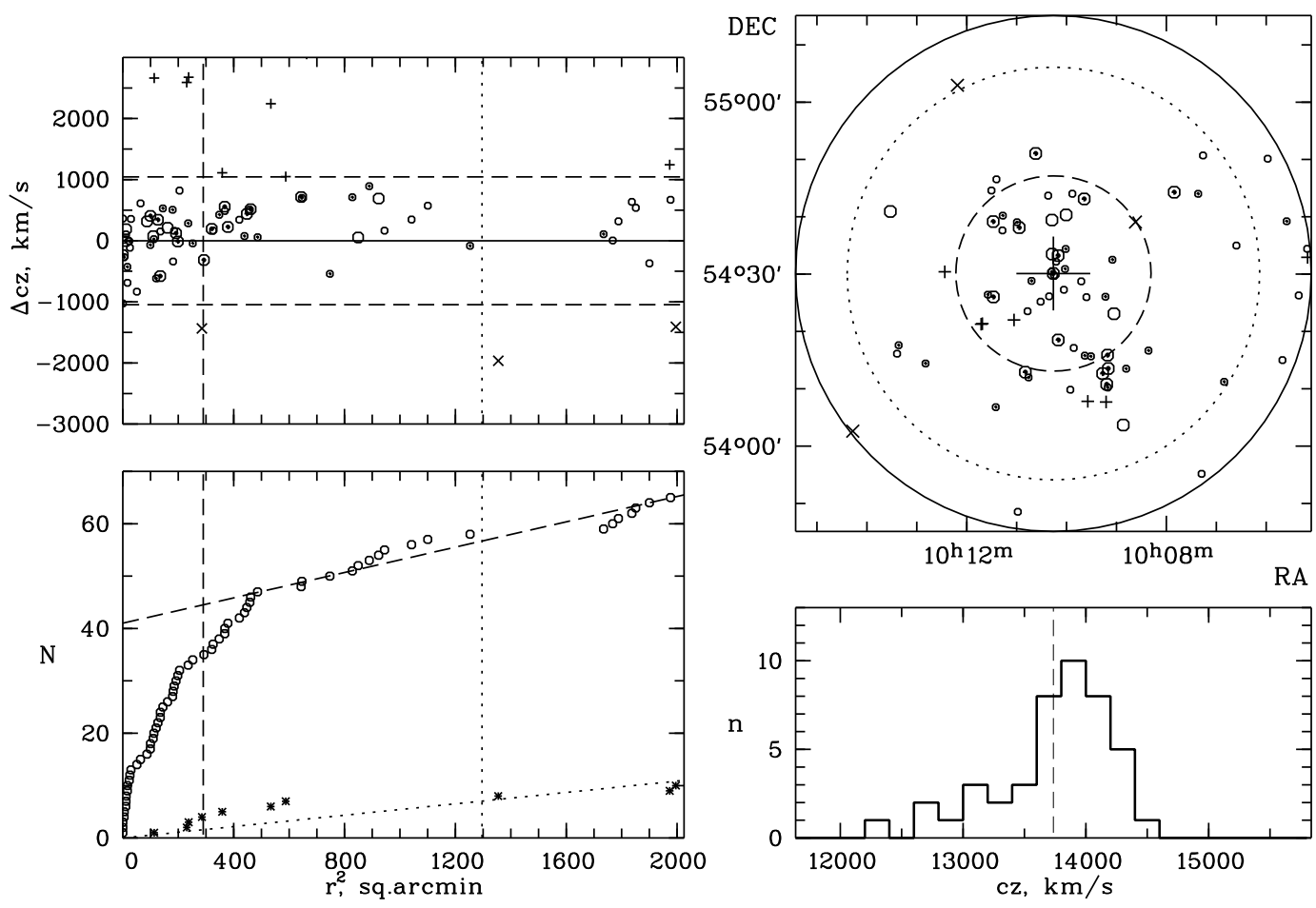

Figure 19. Distribution of galaxies in RXCJ1010. 

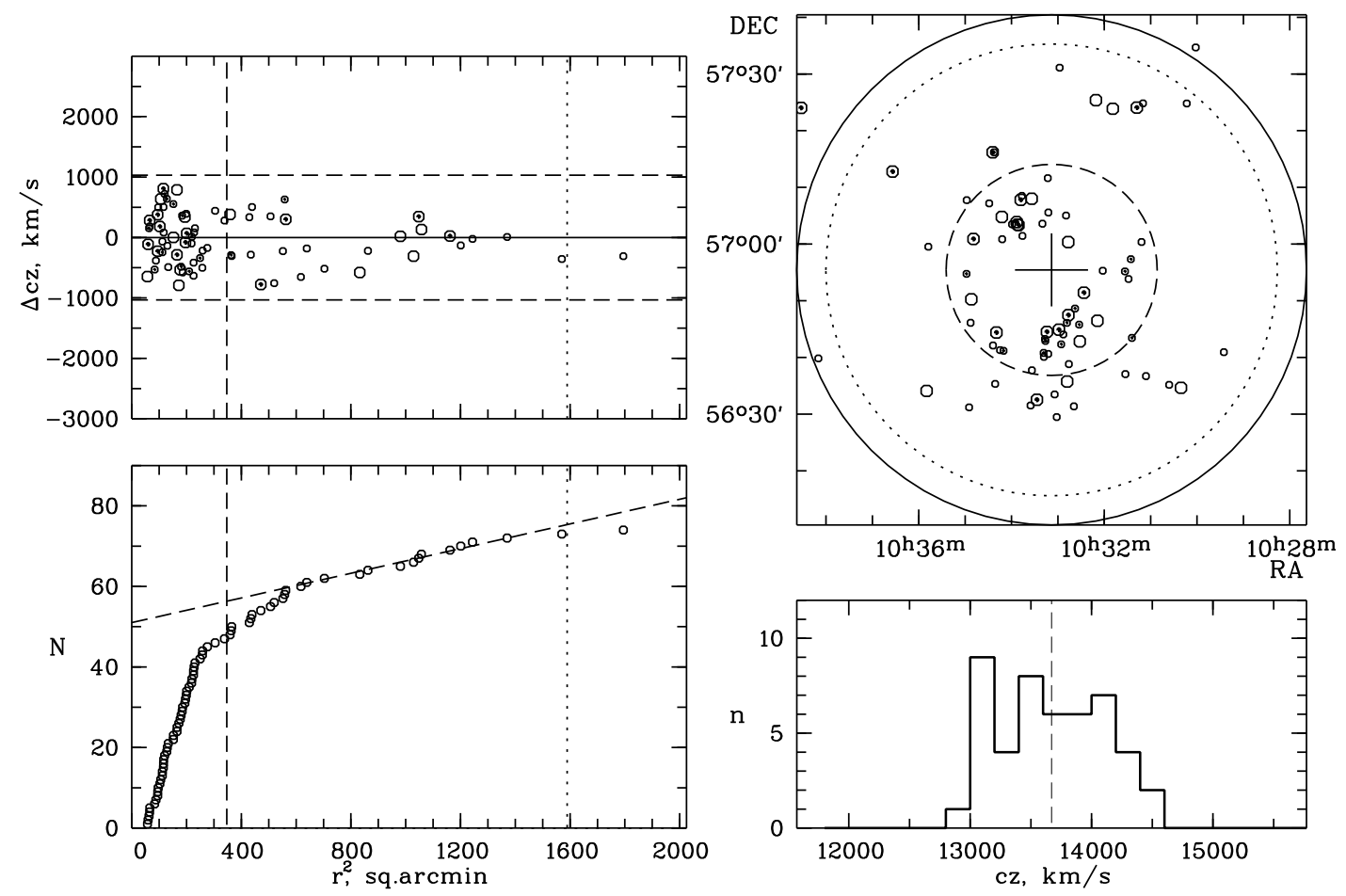

Figure 20. Distribution of galaxies in RXJ1033.
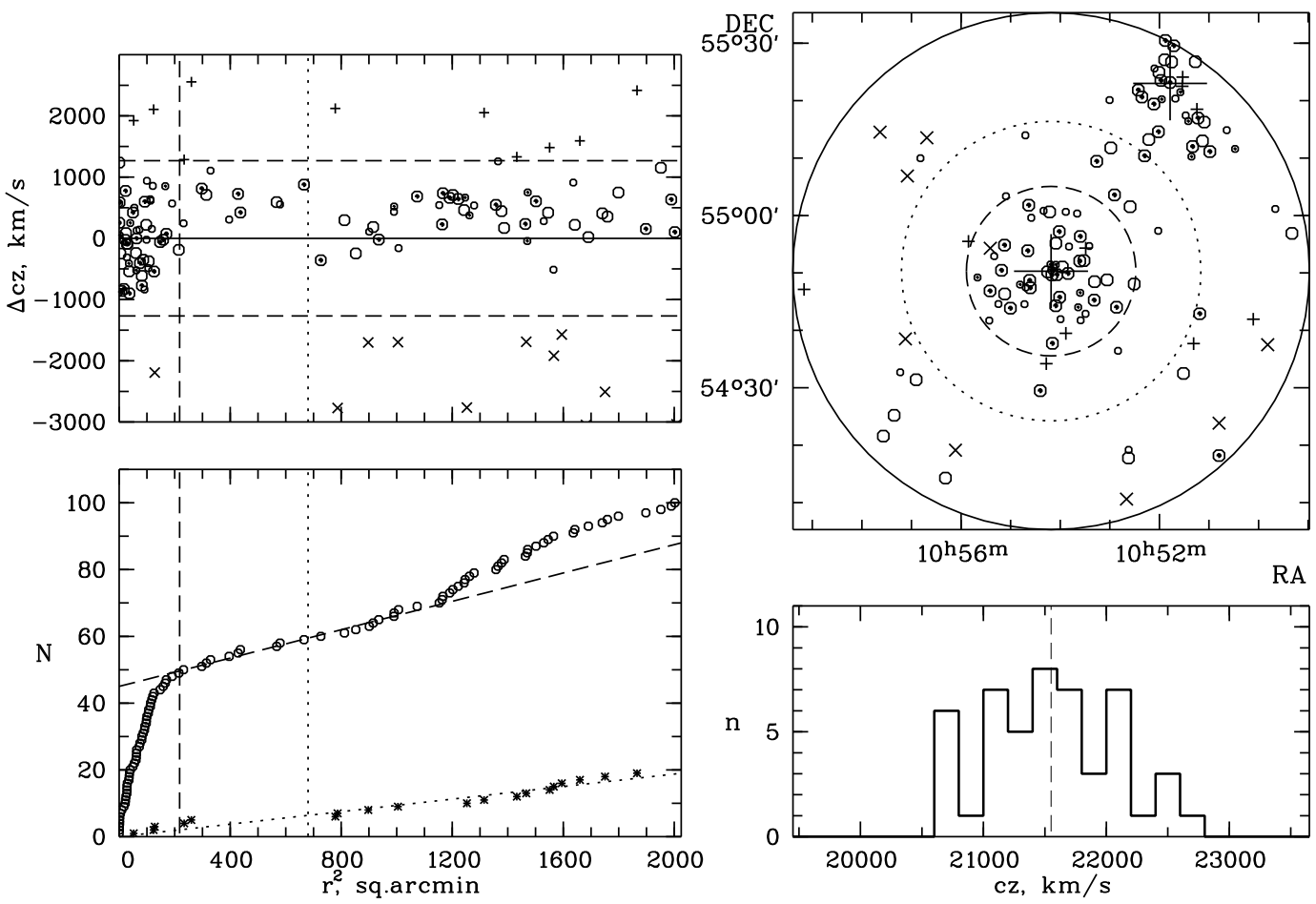

Figure 21. Distribution of galaxies in RXCJ1053A (RXCJ1053B is located at the top right corner and is indicated by a big cross). 

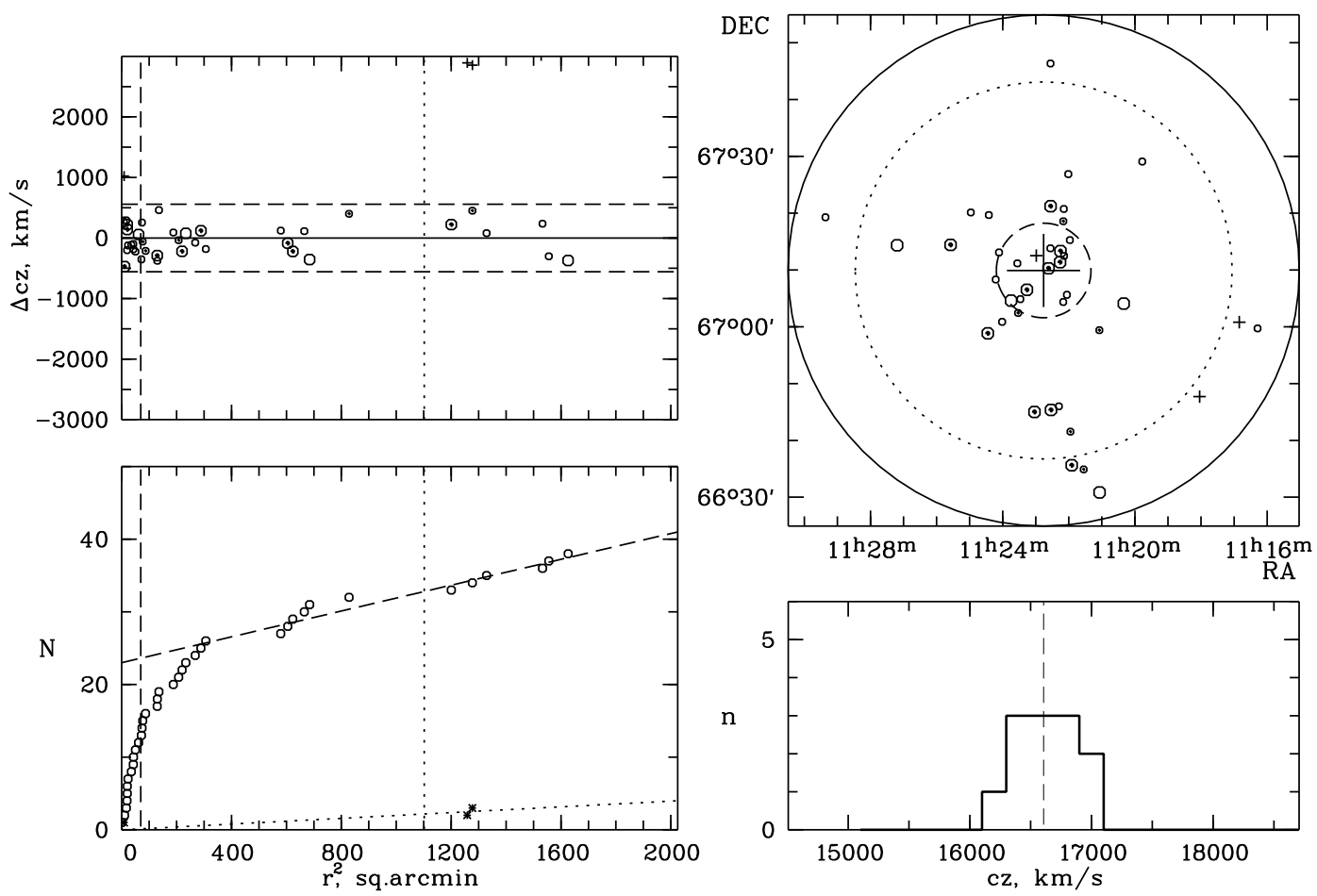

Figure 22. Distribution of galaxies in RXCJ1122.

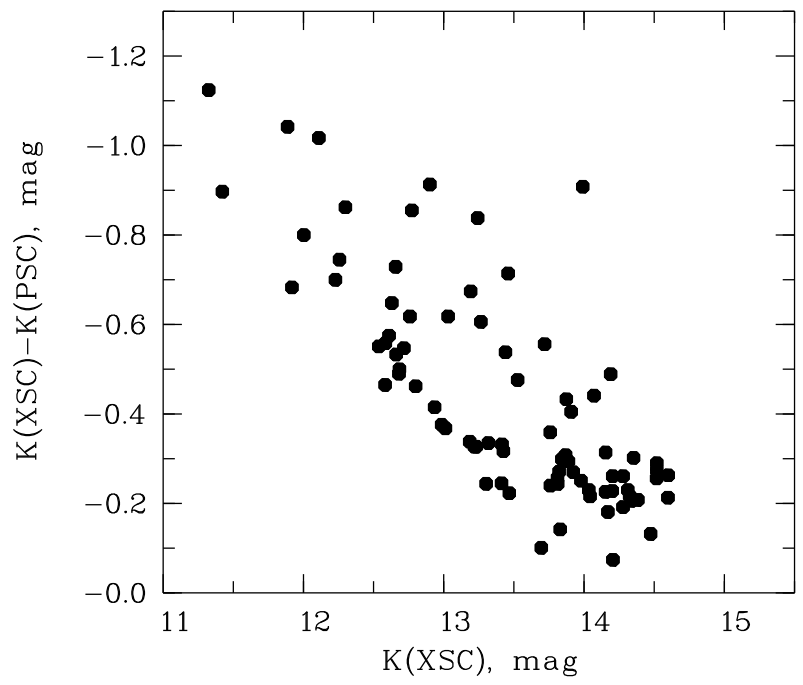

Figure 23. Comparison of the $K$-band magnitudes for the galaxies from the 2MASS extended-source catalog XSC (magnitudes within the $\mu_{K}=20^{m} / \square^{\prime \prime}$ surface-brightness isophote) and the 2MASS point-source catalog PSC (magnitude in the standard aperture) for the A1377 cluster. 
cluster:

$$
\phi^{*}=N_{o b s} / \int_{L_{K, l i m} / L_{K}^{*}}^{+\infty} t^{\alpha} e^{-t} d t
$$

where $t=L / L_{K}^{*}$. We then summed up the luminosities of galaxies down to the limiting magnitude of $15^{m}$ and used the normalization quantity obtained to extrapolate the LF downward to the limiting magnitude of $-21^{m}$, which corresponds to the luminosity $L_{K, \min }$ :

$$
L_{K}=\sum_{i=1}^{N_{o b s}} L_{K, i}+\phi^{*} L_{K}^{*} \int_{L_{K, \min } / L_{K}^{*}}^{L_{K, l i m} / L_{K}^{*}} t^{\alpha+1} e^{-t} d t .
$$

This procedure increases the luminosity of the cluster by $5 \%$ on the average.

In the second variant of the computation of the total luminosities of clusters we searched for the parameters of the Schechter function for composite luminosity function of our sample. To perform this, we counted galaxies in each cluster within $0.5^{m}$-wide magnitude intervals. We then constructed the composite luminosity functions for the virialized regions of UMa clusters and for the clusters located in less densely populated immediate environments using the method described by Colless [18] and approximated the resulting composite LFs via nonlinear least squares method by the Schechter functions [19] with the parameters $M_{K}^{*}=-24.33^{m} \pm 0.04, \alpha=-0.82 \pm 0.02$ and $M_{K}^{*}=-24.20^{m} \pm 0.13, \alpha=-0.92 \pm 0.09$ for UMa clusters and for the clusters from the UMa neighborhood, respectively (we chose our last data point in the domain of faint magnitudes to be $-23.0^{m}$ in order to avoid data incompleteness for more distant clusters). We compute the errors as $\sqrt{d N}$, where $d N$ is the number of galaxies in the $d M$ interval. The exclusion of the highestluminosity data point from the fit has no significant effect on the parameters above. Figure 25 shows both luminosity functions. The parameters of two functions are almost the same and we therefore constructed the composite luminosity function for all clusters and used the Schechter function parameters inferred for the combined sample $\left(M_{K}^{*}=-24.29^{m} \pm 0.05, \alpha=-0.85 \pm 0.03\right)$ to compute the total cluster luminosities listed in Table 2. The total luminosities of clusters of galaxies, calculated using this way, on the average are higher than the corresponding luminosities computed in the first variant by $3 \%$, implying an increase of the mass-to-luminosity ratio in the same proportion. The parameters of the luminosity functions for individual clusters differ by less than $3 \sigma$ from the values above.

Unlike the other authors, we found that parameter $M_{K}^{*}$ in virialized regions of galaxies clusters located in the UMa region is $0.15^{m}$ lower than the same for field galaxies [16]. In the virialized regions of nine nearby rich $(495 \mathrm{~km} / \mathrm{s}<\sigma<1042 \mathrm{~km} / \mathrm{s})$ and X-ray luminous clusters of galaxies the 
Table 2. Properties of clusters in the near IR

\begin{tabular}{l|c|c|c|l}
\hline Cluster & $L_{K, 200}$ & $M / L_{K}$ & $N\left(-21^{m}\right)$ & $f_{E}\left(N_{E}\right)$ \\
\hline A1270 & $5.12 \pm 0.16$ & $55 \pm 18$ & 92 & $0.63(22)$ \\
A1291A & $1.82 \pm 0.10$ & $55 \pm 30$ & 41 & $0.85(11)$ \\
A1291B & $1.78 \pm 0.12$ & $158 \pm 80$ & 50 & $0.69(9)$ \\
A1318 & $2.90 \pm 0.17$ & $36 \pm 17$ & 53 & $0.78(11)$ \\
A1377 & $6.52 \pm 0.15$ & $66 \pm 21$ & 103 & $0.65(26)$ \\
A1383 & $3.76 \pm 0.13$ & $45 \pm 16$ & 72 & $0.67(20)$ \\
A1436 & $6.74 \pm 0.16$ & $79 \pm 23$ & 130 & $0.82(37)$ \\
Anon1 & $4.57 \pm 0.14$ & $83 \pm 28$ & 84 & $0.71(27)$ \\
Anon2 & $1.96 \pm 0.16$ & $14 \pm 8$ & 23 & $0.91(10)$ \\
Anon3 & $1.88 \pm 0.16$ & $47 \pm 23$ & 35 & $0.78(11)$ \\
Anon4 & $2.36 \pm 0.14$ & $44 \pm 20$ & 33 & $0.50(8)$ \\
Sh166 & $1.35 \pm 0.09$ & $44 \pm 23$ & 36 & $0.70(7)$ \\
A1003 & $2.82 \pm 0.16$ & $106 \pm 55$ & 41 & $0.47(8)$ \\
A1169 & $4.25 \pm 0.11$ & $78 \pm 27$ & 89 & $0.68(19)$ \\
A1279 & $0.64 \pm 0.14$ & $17 \pm 16$ & 8 & $0.25(1)$ \\
A1452 & $2.08 \pm 0.09$ & $109 \pm 64$ & 29 & $0.67(8)$ \\
A1461 & $0.82 \pm 0.10$ & $66 \pm 49$ & 15 & $0.75(3)$ \\
A1507 & $2.63 \pm 0.14$ & $52 \pm 21$ & 50 & $0.71(12)$ \\
A1534 & $2.29 \pm 0.14$ & $21 \pm 12$ & 29 & $0.69(9)$ \\
RXCJ1010 & $2.00 \pm 0.16$ & $62 \pm 23$ & 39 & $0.64(7)$ \\
RXJ1033 & $2.69 \pm 0.18$ & $45 \pm 18$ & 52 & $0.56(9)$ \\
RXCJ1053A & $3.94 \pm 0.11$ & $55 \pm 21$ & 80 & $0.68(19)$ \\
RXCJ1053B & $2.59 \pm 0.08$ & $47 \pm 35$ & 46 & $0.58(11)$ \\
RXCJ1122 & $0.79 \pm 0.13$ & $24 \pm 18$ & 16 & $0.80(4)$ \\
\hline
\end{tabular}

$M_{K}^{*}$ parameter is lower by $0.62^{m}[12]$ and the fraction of faint galaxies is much higher $(\alpha=-1.35)$. Rines et al. [12] used the total extrapolated magnitudes.

\section{PROPERTIES OF EARLY-TYPE GALAXIES IN THE VIRIALIZED REGIONS OF CLUSTERS OF GALAXIES}

The fraction of early-type galaxies in clusters is known to increase with increasing local galaxy density [20,21], and so does the luminosity of their spheroidal components [20]. In this paper we 


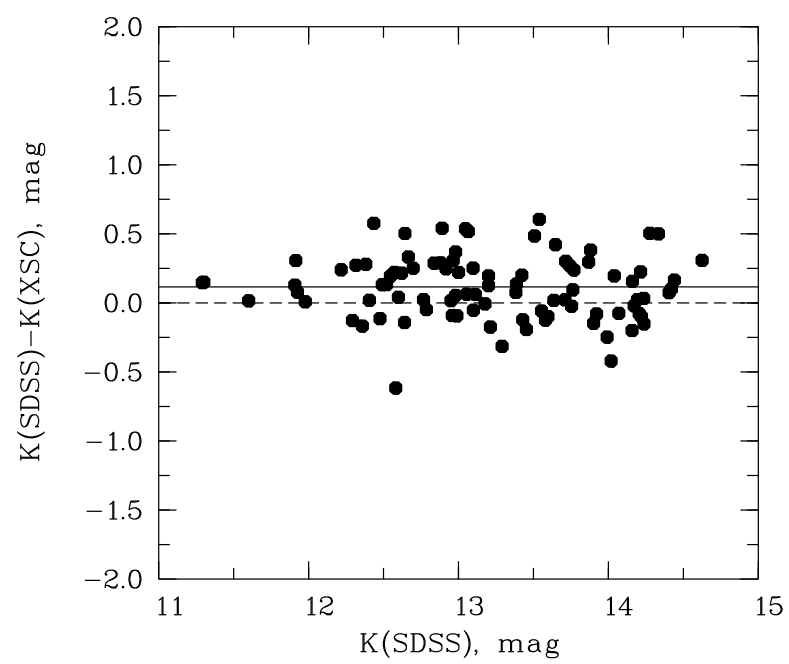

Figure 24. Comparison of the $K$-band magnitudes computed using the $u-r$ color index from the SDSS catalog with the 2 MASS XCS $\mu_{K}=20^{m} / \square^{\prime \prime}$ isophotal magnitudes for galaxies of the A1377 cluster. The solid line indicates the average magnitude difference.

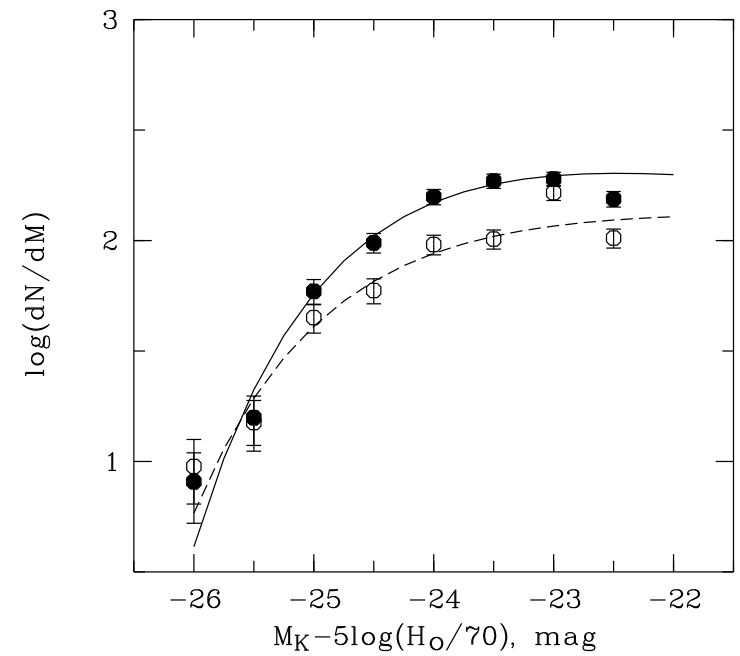

Figure 25. Combined luminosity functions for UMa galaxies (the filled circles) and for field galaxies (the open circles). The solid and dashed lines indicate the corresponding Schechter functions.

analyze the properties of early-type galaxies located in the UMa supercluster and in its immediate vicinity. We used the following criteria to select early-type galaxies (in the $r$-band filter of SDSS): fracDeV $\geq 0.8$ (this parameter characterizes the contribution of the de Vaucouleurs profile to the surface-brightness profile of the galaxy); $R_{90}^{r} / R_{50}^{r} \geq 2.6$ (the concentration index is equal to the ratio of the radii containing $90 \%$ and $50 \%$ of the Petrosian flux). We constructed the composite $K$-band luminosity functions separately for early- and late-type galaxies (Figs. 26 and 27) in the 
virialized regions of clusters of galaxies. We derived the following parameters for the Schechter function:

(a) $M_{K}^{*}=-24.54^{m} \pm 0.18, \alpha=-0.61 \pm 0.09$ for early-type galaxies of the UMa system and $M_{K}^{*}=-24.48^{m} \pm 0.07, \alpha=-0.65 \pm 0.04$ for galaxies in the UMa neighborhood;

(b) $M_{K}^{*}=-24.06^{m} \pm 0.06, \alpha=-1.22 \pm 0.06$ for late-type galaxies of the UMa system and $M_{K}^{*}=-24.05^{m} \pm 0.18, \alpha=-1.23 \pm 0.18$ for galaxies in the UMa neighborhood.

The number of early-type galaxies in 10 UMa clusters is $40 \%$ greater than the same in 11 clusters in the nearest neighborhood of the supercluster, and the number of late-type galaxies is $15 \%$ greater down to the adopted limiting magnitude of $\left(-21^{m}\right)$. We compared the $M_{K}^{*}$ for early-type galaxies in the UMa region $\left(-24.54^{m},-24.48^{m}\right)$ with the similar values for field galaxies [16] $\left(-24.28^{m}\right)$. We performed a similar comparison of the $M_{K}^{*}$ magnitudes for late-type galaxies in the UMa neighborhood $\left(-24.06^{m},-24.05^{m}\right)$ and for field galaxies $\left(-23.73^{m}\right)$ from the same paper. We found that the $M_{K}^{*}$ magnitudes in the virialized regions of clusters of galaxies to be $0.23^{m}$ and $0.32^{m}$ lower for early- and late-type galaxies, respectively, located in the UMa region than the corresponding magnitudes for field galaxies. Moreover, the number of early-type galaxies in the clusters located in the region studied decreases $(\alpha>-1)$ and that of late-type galaxies increases $(\alpha<-1)$ with decreasing galaxy luminosity.

We calculate the fraction of early-type galaxies (i.e., the ratio of number of early-type galaxies to the total number of galaxies) inside $R_{200}$ down to a fixed limiting magnitude corresponding to $M_{K}^{*}+1$, where $M_{K}^{*}$ is equal to the characteristic magnitude of the cluster sample $\left(-24.29^{m}\right)$. We found that, on the average, the fraction of bright early-type galaxies in the UMa system is equal to $0.71 \pm 0.03$ (without A1291B and Anon2), and the corresponding fraction for the neighborhood of the UMa supercluster is $0.66 \pm 0.03$ (without A1279). Figure 28 presents the fraction of bright early-type galaxies plotted as a function of the cluster mass. It is evident from the figure that the fraction of early-type galaxies remains almost unchanged with the cluster mass lying in the interval $10^{13} M_{\odot}<M<5.0 \times 10^{14} M_{\odot}$. A similar result for clusters of galaxies was obtained, e.g., by Balogh et al. [22] and Tanaka et al. [23]. At the same time, Martinez et al. [24] and Weinmann et al. [25] found that the fraction of such galaxies in groups decreases with the decreasing mass of the group. A1279 and Anon2 with their extremely large early-type galaxy populations stand out among the clusters of galaxies studied in this paper and are marked by plus signs in Fig. 28.

We found the average parameters of bright early-type galaxies (down to the fixed magnitude of $\left.M_{K}^{*}+1\right)$, the $K$-band absolute magnitude, the $g-r$ and $u-r$ color indices, the effective bulge 
Table 3. Average parameters for early-type galaxies $\left(M_{K}<-23.29^{m}\right)$ and their dispersions

\begin{tabular}{c|r|c|c|c|c|c|c|c|l}
\hline & $N_{c}$ & $N_{g}$ & $\begin{array}{c}M_{K}, \\
\mathrm{mag}\end{array}$ & $\begin{array}{c}g-r, \\
\mathrm{mag}\end{array}$ & $\begin{array}{c}u-r, \\
\mathrm{mag}\end{array}$ & $\begin{array}{c}r_{e}, \\
\mathrm{kpc}\end{array}$ & $b / a$ & fracDeV & $R_{90}^{r} / R_{50}^{r}$ \\
\hline UMa & 10 & 180 & $-24.19(0.59)$ & $0.85(0.04)$ & $2.71(0.16)$ & $4.12(1.62)$ & $0.69(0.18)$ & $0.97(0.06)$ & $3.05(0.24)$ \\
field & 11 & 109 & $-24.32(0.68)$ & $0.86(0.04)$ & $2.73(0.12)$ & $4.41(2.17)$ & $0.69(0.19)$ & $0.97(0.05)$ & $3.02(0.22)$ \\
I & 5 & 117 & $-24.20(0.60)$ & $0.85(0.04)$ & $2.70(0.14)$ & $4.41(1.64)$ & $0.70(0.18)$ & $0.97(0.06)$ & $3.02(0.23)$ \\
II & 11 & 138 & $-24.25(0.65)$ & $0.86(0.04)$ & $2.72(0.15)$ & $4.06(1.94)$ & $0.67(0.18)$ & $0.97(0.05)$ & $3.05(0.24)$ \\
III & 5 & 34 & $-24.32(0.64)$ & $0.86(0.04)$ & $2.75(0.14)$ & $4.60(1.95)$ & $0.72(0.20)$ & $0.97(0.05)$ & $3.09(0.23)$ \\
\hline
\end{tabular}

radius in the $r$ band, the axial ratio $b / a$, the contribution of the de Vaucouleurs bulge to the surfacebrightness profile and to the concentration index, which is equal to the ratio of the galactocentric radii containing $50 \%$ and $90 \%$ of the Petrosian flux. Table 3 lists the data obtained for the UMa supercluster, its immediate vicinity, and three samples of galaxies. Cluster subsamples are selected from the list of clusters studied, which subdivided by mass:

(I) $\mathrm{M}>3 \times 10^{14} M_{\odot}$;

(II) $1 \times 10^{14} M_{\odot}<\mathrm{M}<3 \times 10^{14} M_{\odot} ;$

(III) $\mathrm{M}<1 \times 10^{14} M_{\odot}$.

Sample I consists of the most massive clusters: A1003, A1169, A1377, A1436, and Anon1; sample II consists of A1270, A1291A, A1318, A1383, A1452, A1507, Anon4, RXCJ1010, RXJ1033, RXCJ1053A, and RXCJ1053B, and sample III consists of A1461, A1534, Anon3, Sh166, and RXCJ1122. An analysis of the data listed in Table 3 leads us to conclude that early-type (i.e., bulge dominated) galaxies have nearly the same properties in all samples. There is a weak tendency for these galaxies to be brighter in the IR on the one hand, in field clusters in the vicinity of UMa, and, on the other hand, in less massive clusters (i.e., in those with lower velocity dispersion).

\section{THE RELATION BETWEEN THE TOTAL $K$-BAND LUMINOSITIES AND MASSES OF CLUSTERS}

The measurements of the $K$-band luminosities of clusters and groups of galaxies (mostly of the virialized regions) [6, 10-12] showed that the mass-to-luminosity ratio and the luminosities of clusters increase with increasing mass of the system (the mass of the dark halo). In this paper we find the relations between these parameters for the particular region: the UMa supercluster and its 


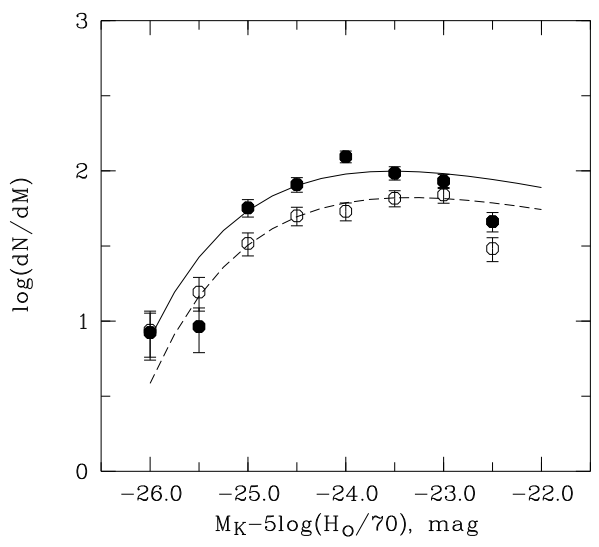

Figure 26. Composite luminosity functions for early-type galaxies. Same designations as in Fig. 25.

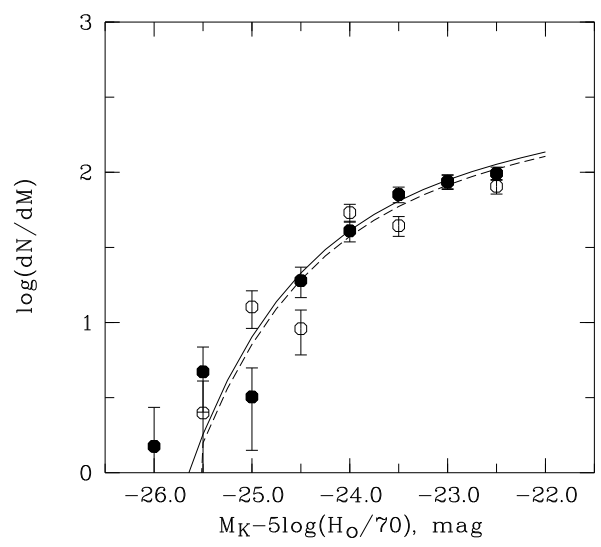

Figure 27. Composite luminosity functions for late-type galaxies. Same designations as in Fig. 25.

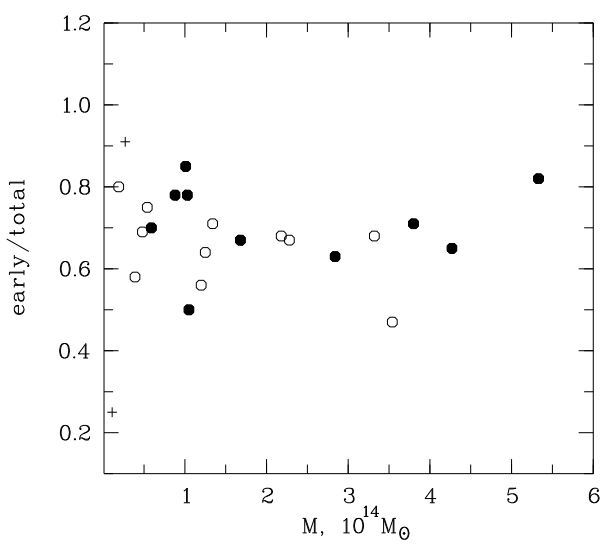

Figure 28. The fraction of early-type galaxies among the galaxies brighter than $M_{K}^{*}+1$ as a function of the cluster mass inside $R_{200}$. The filled and open circles correspond to the clusters located in the UMa and in its neighborhood, respectively. The plus signs indicate the clusters with the smallest (A1279) and greatest (Anon2) fractions of early-type galaxies. 
nearest neighborhood. As we already pointed out above, we find the total luminosities of clusters of galaxies using the Schechter parameters for the composite luminosity function $\left(-24.29^{m},-0.85\right)$. The relations between the cluster mass and $K$-band luminosity for the UMa supercluster and field clusters in its immediate vicinity have the following form (in logarithmic form):

$$
\begin{aligned}
& \log L_{K, 200}=0.75( \pm 0.10) \log M_{200}+1.80( \pm 1.22) \\
& \log L_{K, 200}=0.63( \pm 0.18) \log M_{200}+3.50( \pm 2.36)
\end{aligned}
$$

We present these relations in Fig. 29. The relations obtained are averages of direct and inverse regression derived by treating luminosity and mass, respectively, as independent variables. The rms scatter is equal to 0.08 and 0.16 for the relations derived for UMa system and field clusters, respectively. The scatter in the supercluster is about twice smaller than in the field. Note that the slope of the relation between $M_{200}$ and $L_{K, 200}$ for clusters of galaxies in the UMa coincides within the quoted errors with the corresponding slope for field clusters. The luminosities of clusters of galaxies are computed in projection, i.e., within cylinders, and deprojection may decrease them by about $20 \%$ (depending on the location of the cluster). The luminosity corrections should be minimal, because the filamentary structures in the supercluster are aligned parallel to the sky plane (see [6, Fig. 2]). A comparison shows that despite of use of different techniques our results agree with those obtained by other authors: $L_{K} \propto M^{0.72 \pm 0.04}[10]$ and $L_{K} \propto M^{0.64 \pm 0.06}$ [11]. The correlation between the masses and luminosities of clusters of galaxies allows the masses of individual clusters to be estimated from their $K$-band luminosities. The scatter of this relation is of special interest. This is believed to be most likely due to the deviation of the dynamical state of the cluster from virial equilibrium (see, e.g., [11]). In our sample the clusters those with the greatest deviations from the mean value are Anon2 and A1291B from UMa and A1279, which a very poor cluster located in the UMa neighborhood. We did not use them for computing our regression models. The A1003, A1452, and A1461 clusters in the UMa neighborhood also deviate from the average relation due to their higher mass (dispersion), which appears to correspond to non-equilibrium state, and the A1534 cluster-due to its lower mass. The relation between the mass and the total luminosity can actually be used to identify clusters of galaxies that are in a peculiar state, with either too high or too low velocity (mass) dispersion combined with rather high luminosity. The rather small scatter that we obtained for the clusters belonging to the UMa supercluster (which is the densest part of the system of clusters) and which exceeds the scatter obtained in other similar studies based on various samples of clusters, which do not form a supercluster, may be indicative of the synchronous nature of the evolution of clusters within a single system (UMa). We use the same procedures as in case of the relation between $L_{K, 200}$ and $M_{200}$ to derive a formula to connect the logarithms of $M_{200} / L_{K, 200}$ and $M_{200}$ for the UMa supercluster and for the clusters located in the UMa field. The 
resulting relations have the following form:

$$
\begin{aligned}
& \log M_{200} / L_{K, 200}=0.36( \pm 0.15) \log M_{200}-3.49( \pm 0.96), \\
& \log M_{200} / L_{K, 200}=0.60( \pm 0.18) \log M_{200}-6.75( \pm 1.01)
\end{aligned}
$$

and are shown in Fig. 30. The scatter is the same as that of the relations between the mass and luminosity. It is interesting that the slope of these relations is higher by about one third than the slopes of the relations $M / L \propto M^{0.25}$ and $M / L \propto M^{0.37}$ derived from the relations between $L_{K, 200}$ and $M_{200}$ given above. Moreover, the slope of the dependence of $M / L_{K}$ on $M$ for clusters in the UMa neighborhood is somewhat steeper than that for UMa clusters and is equal to the corresponding slope for groups of galaxies given below. Various authors obtained the following results for other samples of clusters of galaxies: $M / L_{K} \propto M^{0.26 \pm 0.04}$ [10] and $M / L_{K} \propto M^{0.31 \pm 0.09}$ [12], but $M / L_{K} \propto M^{0.56 \pm 0.05}$ [11] for groups of galaxies. The average $M / L$ ratio inside $R_{200}$ for the UMa supercluster is equal to $55 \pm 5 M_{\odot} / L_{\odot}$, and the corresponding ratio for field clusters in the UMa neighborhood is $60 \pm 8 M_{\odot} / L \odot$. Note for comparison, that the average $M / L$ ratio inside $R_{200}$ for rich clusters of galaxies (with the masses estimated using the method of caustics in spheres and the luminosities computed inside the cylinders, like in this paper) from [12] is $49 \pm 5 M_{\odot} / L_{\odot}$, which agrees with our result within the quoted errors. The existence of the $L-M$ and $M / L-M$ relations indicates that the formation of stars or galaxies in clusters and groups are regular processes, however, the efficiency of star formation inside virialized regions of the clusters may be a decreasing function of the cluster mass (see, e.g., [10]), as evidenced by the smaller-than-unity slope in Fig. 29. Note that the unaccounted contribution of the radiation from intragalactic and intracluster stars is an increasing function of mass and may amount to $5-50 \%$ (see, e.g.,[12]).

\subsection{Number of Galaxies in Clusters}

Clusters of galaxies are massive dark halos where baryonic matter in the form of gas and galaxies is located in the places of highest concentration. The number of galaxies down to a certain limiting magnitude located in the cluster inside a certain radius (the halo occupation number) is the main parameter, which can be used to compare the results of model computations with observations. To compute the number of galaxies in a cluster located within $R_{200}$, we adopted the Schechter function parameters for the composite luminosity function $\left(-24.29^{m},-0.85\right)$, extrapolated this function down to a certain limiting magnitude equal to $-21^{m}$. The normalization factor is equal to the number of observed galaxies down to the limiting magnitude of $15^{m}$. We derived the relations between the 
logarithms of $N_{200}$ and $M_{200}$ for the UMa supercluster and for clusters in the UMa field using the same procedures as those employed to derive the previous relations. Figure 31 presents the resulting relations, which have the following form:

$$
\begin{aligned}
& \log N_{200}=0.67( \pm 0.10) \log M_{200}-7.75( \pm 0.65), \\
& \log N_{200}=0.67( \pm 0.21) \log M_{200}-7.80( \pm 1.21) .
\end{aligned}
$$

The scatter is the same as in case of the previous relations-it is twice smaller for UMa clusters compared to the clusters located in the immediate neighborhood of the supercluster. The slope of the relation between $N$ and $M$ is the same for both subsamples in what appears to be consistent with the results of some studies while differing slightly from those of other investigations (possibly, due to the methods used to estimate the errors). For example, the following relations were derived for a sample of rich clusters: $N \propto M^{0.70 \pm 0.09}$ [12] for $R_{200}$ and $N \propto M^{0.84 \pm 0.04}$ [10] for $R_{500}$. For groups of galaxies within $R_{200}$ the relation $N \propto M^{0.56 \pm 0.05}$ [11] was derived. From the theoretical viewpoint the slope must be smaller than unity (it must lie in the $0.56-0.74$ interval), i.e., the efficiency of the formation of galaxies must be lower and/or the processes of galaxy disruption must be less efficient in more massive halos (see, e.g., [12] and references therein). We list the inferred parameters of clusters of galaxies in Table 2. The number $N(-21)$ of galaxies in the cluster is equal to the number of galaxies down to a limiting magnitude of $-21^{m}$. We determine the error of $L_{K}$ by subtracting the luminosity of each galaxy (one after another) from the total luminosity of the cluster and averaging the resulting deviations. In the Table $2 f_{E}$ denotes the fraction of early-type galaxies down to a limiting magnitude of $M_{K}^{*}+1$ and the $N_{E}$ is the number of such galaxies and is given in the parentheses.

\section{CONCLUSIONS}

The Ursa Major supercluster is a layered system consisting of large filamentary structures (see [5, Figs. 1a-1c]) with the mean redshifts of 0.051, 0.061, and 0.071. Only three clusters in this system, A1377, A1436, and Anon1, are sufficiently rich both in terms of the number of galaxies inside the virial radius and in terms of X-ray luminosity (Table 1). UMa is nevertheless a supercluster of special interest, because it is a rather isolated system (see [5, Figs. 1a-1c]) with no rich X-ray clusters of galaxies or other superclusters in its vicinity, i.e., UMa is located in the region of the Universe with a low density of clusters of galaxies [26]. One may say that the UMa supercluster is located in a lower-than-average density region and such a location must have an effect on 


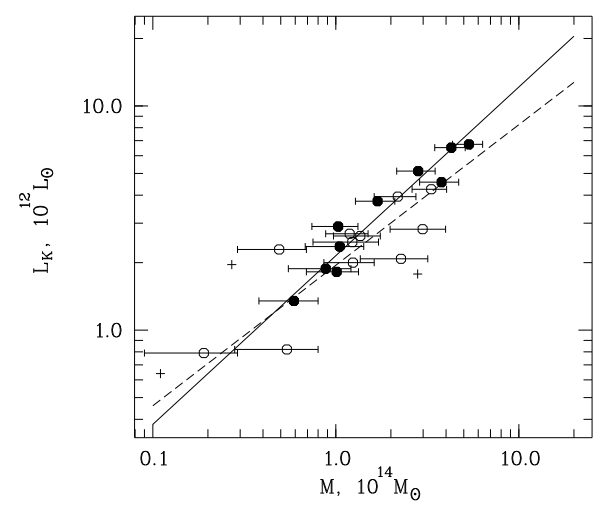

Figure 29. Total luminosity of galaxies $\left(M_{K}^{*}<-21^{m}\right)$ as a function of the cluster mass inside $R_{200}$. The filled and open circles correspond to UMa and field clusters, respectively. The plus signs indicate the clusters A1279, Anon2, and A1291B, which were not used to derive the regression relations. These relations for UMa and field clusters are shown by the solid and dashed lines, respectively.

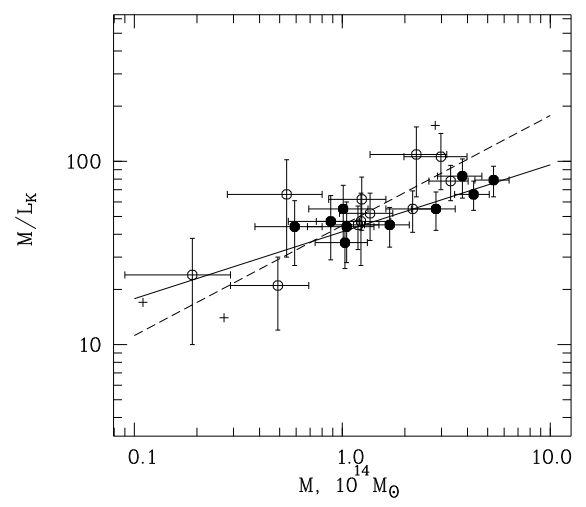

Figure 30. The mass-to-light ratio $\left(M_{K}^{*}<-21^{m}\right)$ as a function of the cluster mass inside $R_{200}$. Designations are the same as in Fig. 29.

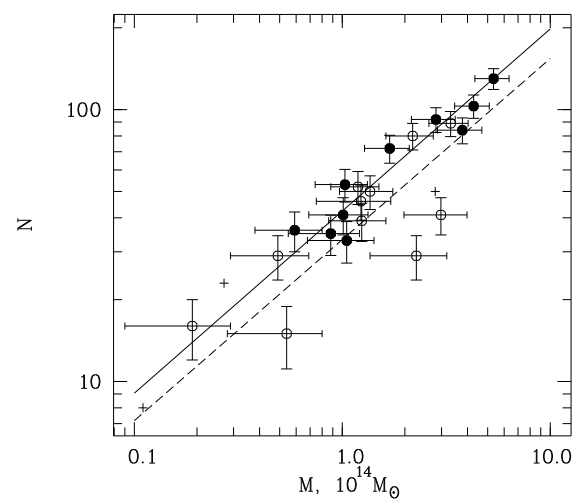

Figure 31. The number of galaxies in the cluster $\left(M_{K}^{*}<-21^{m}\right)$ as a function of the cluster mass inside $R_{200}$. Designations are the same as in Fig. 29. 
the entire system of clusters as a whole. The dynamic evolution in the regions of high density of galaxies, such as superclusters, is believed to begin very early and continue until the present time (see, e.g., [27]). In this paper we study and compare some of the properties of virialized regions of clusters of galaxies located in the vicinity of the UMa supercluster, both in its central part with a factor-of-three galaxy overdensity, and in its periphery with radius of $75 \mathrm{Mpc}$. In this study we use the data of the SDSS and 2MASS catalogs. Below we compare our results for two samples of clusters.

(1) The Schechter functions for the composite luminosity functions of virialized regions of clusters of galaxies have similar parameters $\left(M_{K}^{*}, \alpha\right)$ in UMa and in its neighborhood, and $M_{K}^{*}$ for the composite LF is $0.15^{m}$ lower than the corresponding parameter for the LF of field clusters [16].

(2) The Schechter functions for the composite luminosity functions of early- and late-type galaxies in virialized regions of clusters also have similar $\left(M_{K}^{*}, \alpha\right)$ parameters in UMa and in its vicinity, and their $M_{K}^{*}$ are lower than the corresponding parameter for field galaxies [16]. The fraction of early-type galaxies is higher by $40 \%$ in UMa than in clusters located in its vicinity. The number of spiral galaxies in UMa is greater by $15 \%$, and the number of such galaxies increases $(\alpha>1)$, whereas that of early-type galaxies decreases $(\alpha<1)$ with increasing galaxy magnitude.

(3) The fraction of early-type galaxies down to a limiting magnitude of $M_{K}^{*}+1$, which is equal to -23.29 , is of about $70 \%$ in UMa clusters and in the clusters located in the UMa neighborhood. This fraction does not increase with increasing cluster mass in the cluster mass interval $10^{13} M_{\odot}<$ $\mathrm{M}<5.0 \times 10^{14} M_{\odot}$. The average parameters of early-type galaxies are almost the same for clusters located in the central dense region of UMa and in its nearest vicinity, as well as in poor and rich clusters.

(4) The main parameters of clusters inside $R_{200}$ in the UMa supercluster region $\left(L_{K, 200}\right.$, $M_{200} / L_{K, 200}$, and $\left.N_{200}\right)$ increase with the mass of the system. The forms of the relations between these parameters agree within the errors with the relations obtained for other samples of clusters of galaxies drawn with no regard to the possible membership in superclusters. The clusters located in the densest region and identified as the UMa supercluster exhibit twice smaller scatter in all relations compared to the corresponding scatter for field clusters located within $75 \mathrm{Mpc}$ of the supercluster. 


\section{ACKNOWLEDGMENTS}

This work was supported by the Russian Foundation for Basic Research (grant no. 07-02$01417 a)$.

This research has made use the NASA/IPAC Extragalactic Database (NED, http://nedwww.ipac.caltech.edu/), which is operated by the Jet Propulsion Laboratory, California Institute of Technology, under contract with the NASA; Sloan Digital Sky Survey (SDSS, http://www.sdss.org/); data products from the Two Micron All Sky Survey (2MASS, http://www.ipac.caltech.edu/2mass/releases/allsky/, which is a joint project of the University of Massachusetts and Infrared Processing and Analysis Center/Caltech, funded by NASA and NSF, and X-Rays Clusters Database (BAX, http://bax.ast.obs-mip.fr/), which is operated by the Laboratoire d'Astrophysique de Tarbes-Toulouse (LATT), under contract with the Centre National d'Etudes Spatiales (CNES). Funding for the creation and distribution of the SDSS Archive has been provided by the Alfred P. Sloan Foundation, the Participating Institutions, the National Aeronautics and Space Administration, the National Science Foundation, the US Department of Energy, the Japanese Monbukagakusho, and the Max Planck Society. 


\section{References}

1. M. Einasto, J. Einasto, E. Tago, et al., Astronom. and Astrophys. 464, 815 (2007).

2. T. A. Small, L. W. Sargent, and D. Hamilton, Astrophys. J. 487, 512 (1997).

3. C. P. Haines, P. Merluzzi, A. Mercurio, et al., Monthly Notices Roy. Astronom. Soc. 371, 55 (2006).

4. A. Mercurio, P. Merluzzi, C. P. Haines, et al., Monthly Notices Roy. Astronom. Soc. 368, 109 (2006).

5. F. G. Kopylova and A. I. Kopylov, Astron. Lett. 33, 211 (2007).

6. F. G. Kopylova and A. I. Kopylov, Astron. Lett. 32, 84 (2006).

7. M. R. Carlberg, H. K. C. Yee, E. Ellingson, et al., Astrophys. J. Suppl. 485, L13 (1997).

8. R. Sadat, A. Blanchard, J.-P. Kneib, et al., Astronom. and Astrophys. 424, 1097 (2004).

9. J. A. L. Aquerri, R. Sánshez-Janssen and C. Muños-Tuñón, Astronom. and Astrophys. 471, 17 (2007).

10. Y.-T. Lin, J. J. Mohr, and S. A. Stanford, Astrophys. J. 610, 745 (2004).

11. M. Ramella, W. Boschin, M. Geller, et al., Astronom. J. 128, 2022 (2004).

12. K. Rines, M.J. Geller, A. Diaferio, et al., Astronom. J. 128, 1078 (2004).

13. T. H. Jarrett, T. Chester, R. Cutri, et al., Astronom. J. 119, 2498 (2000).

14. M. Obrić, Ž. Ivezić, P. N. Best, et al., Monthly Notices Roy. Astronom. Soc. 370,1677 (2000).

15. A. W. Graham, S. P. Driver, V. Petrosian, et al., Astronom. J. 130, 1535 (2005).

16. C.S. Kochanek, M.A. Pahre, E.E. Falco, et al., Astrophys. J. 560, 566 (2001).

17. M. A. Strauss, D. H. Weinberg, R. H. Lupton, et al., Astronom. J. 124, 1810 (2002).

18. M. Colless, Monthly Notices Roy. Astronom. Soc. 237, 799 (1989).

19. P. Schechter, Astrophys. J. 203, 297 (1976).

20. A. Dressler, Astrophys. J. 236, 351 (1980).

21. T. Goto, C. Yamauchi, Y. Fujita, et al., Monthly Notices Roy. Astronom. Soc. 346, 601 (2003).

22. M. L. Balogh, I. K. Baldry, R. Nicol, et al., Astrophys. J. 615, L101 (2004).

23. M. Tanaka, T. Goto, S. Okamura, et al., Astronom. J. 128, 2677 (2004).

24. H. J. Martinez, A. Zandivarez, M. Domingues, et al., Monthly Notices Roy. Astronom. Soc. 333, L31 (2002).

25. S. M. Weinmann, F. C. van den Bosch, X. Yang, and H. J. Mo, Monthly Notices Roy. Astronom. Soc. 366, 2 (2006).

26. A. V. Tikhonov, A. I. Kopylov, S. Gottlober, and G. Yepes, astro-ph/0805.2270.

27. J. Einasto, E. Tago, M. Einasto, et al., Astronom. and Astrophys. 439, 45 (2005). 